\title{
Nonzero Sum Differential Game of Mean-Field BSDEs with Jumps under Partial Information
}

\author{
Xiaolan Chen ${ }^{1}$ and Qingfeng Zhu ${ }^{1,2}$ \\ ${ }^{1}$ School of Mathematics and Quantitative Economics, Shandong University of Finance and Economics, Jinan 250014, China \\ ${ }^{2}$ Institute for Financial Studies and School of Mathematics, Shandong University, Jinan 250100, China \\ Correspondence should be addressed to Qingfeng Zhu; zhuqf508@sohu.com
}

Received 21 March 2014; Revised 11 May 2014; Accepted 5 June 2014; Published 3 July 2014

Academic Editor: Guangchen Wang

Copyright (c) $2014 \mathrm{X}$. Chen and Q. Zhu. This is an open access article distributed under the Creative Commons Attribution License, which permits unrestricted use, distribution, and reproduction in any medium, provided the original work is properly cited.

\begin{abstract}
This paper is concerned with a kind of nonzero sum differential game of mean-field backward stochastic differential equations with jump (MF-BSDEJ), in which the coefficient contains not only the state process but also its marginal distribution. Moreover, the cost functional is also of mean-field type. It is required that the control is adapted to a subfiltration of the filtration generated by the underlying Brownian motion and Poisson random measure. We establish a necessary condition in the form of maximum principle with Pontryagin's type for open-loop Nash equilibrium point of this type of partial information game and then give a verification theorem which is a sufficient condition for Nash equilibrium point. The theoretical results are applied to study a partial information linear-quadratic (LQ) game.
\end{abstract}

\section{Introduction}

Game theory had been an active area of research and a useful tool in many applications, particularly in biology and economics. The study of differential games was originally stated by Isaacs [1] and then summed up and developed by Basar and Olsder [2], Yeung and Petrosyan [3], and so forth. Berkovitz [4], Fleming [5], Elliott and Kalton [6], and Friedman [7] established the foundations for zero sum differential games and Varaiya [8] and Elliott and Davis [9] for stochastic differential games. Next, the advances in stochastic differential games continue to appear over a large number of fields. Please refer to Hamadène [10], Hamadène et al. [11], Altman [12], Wu and $\mathrm{Yu}$ [13], Yu and Ji [14], and Wang and $\mathrm{Yu}$ [15] for more information.

For the partial information two-person zero sum (or nonzero sum) stochastic differential games, the objective is to find a saddle point (or equilibrium point) for which the controller has less information than the complete information filtration $\left\{\mathscr{F}_{t}\right\}_{t \geq 0}$. Recently, An and Øksendal $[16,17]$ and An et al. [18] established a maximum principle for partial information differential games of stochastic differential equations with jump (SDEJ). Wang and Yu [19] developed some results for optimal control of BSDEs and established a maximum principle for partial information differential games of backward stochastic differential equations (BSDEs). They established a necessary condition in the form of maximum principle with Pontryagin's type for open-loop Nash equilibrium point of this type of partial information game and gave a verification theorem which is a sufficient condition for Nash equilibrium point. Meng and Tang [20] and Hui and Xiao [21] established a maximum principle for differential games of forward-backward SDE under partial information. Øksendal and Sulem [22] established a general maximum principle for forward-backward stochastic differential games for Itô-Lévy processes with partial information and applied the theory to optimal portfolio and consumption problems under model uncertainty, in markets modeled by Itô-Lévy processes.

To the best of our knowledge, there are few results about the partial information differential games of the discontinuous mean-field backward stochastic system. In the present paper we will research this topic. This paper is concerned with a new kind of nonzero sum differential game of meanfield backward stochastic differential equations with jump (MF-BSDEJ) under partial information. It is required that the control is adapted to a subfiltration of the filtration generated 
by the underlying Brownian motion and Poisson random measure. We establish a necessary condition in the form of maximum principle with Pontryagin's type for open-loop Nash equilibrium point of this type of partial information game and then give a verification theorem which is a sufficient condition for Nash equilibrium point. We note that the state system and the cost function in [22] are not mean-field, and the game systems in $[15,19]$ are BSDEs. The theoretical results are applied to study a partial information linear-quadratic (LQ) game.

The rest of this paper is organized as follows. In Section 2, we state our partial information differential game of MFBSDEJ and the main assumptions. Section 3 is devoted to the necessary optimality conditions. In Section 4, we obtain the sufficient maximum principle of differential game of MFBSDEJ under partial information. In Section 5, we give a partial information linear-quadratic (LQ) game as example to show the applications of our theoretical results.

\section{Statement of the Problems}

Let $(\Omega, \mathscr{F}, P)$ be a completed probability space. We suppose that the filtration $\left\{\mathscr{F}_{t}\right\}_{t \geq 0}$ is generated by the following two mutually independent processes: a $d$-dimensional standard Brownian motion $\{B(t)\}_{t \geq 0}$ and a Poisson random measure $N$ on $\mathbb{R}_{+} \times E$, where $E \subset \mathbb{R}^{l}$ is a nonempty open set equipped with its Borel field $\mathscr{B}(E)$, with compensator $\widehat{N}(d e d t)=$ $\pi(d e) d t$, such that $\widetilde{N}(A \times[0, t])=(N-\widehat{N})(A \times[0, t])_{t \geq 0}$ is a martingale for $\forall A \in \mathscr{B}(E)$ satisfying $\pi(A)<\infty$. $\pi$ is assumed to be a $\sigma$-finite measure on $(E, \mathscr{B}(E))$ and called the characteristic measure. Let $\mathscr{N}$ denote the class of $P$-null elements of $\mathscr{F}$. For each $t \in[0, T]$, we define $\mathscr{F}_{t}=\mathscr{F}_{t}^{W} \vee \mathscr{F}_{t}^{N}$, where for any process $\{\eta(t)\}, \mathscr{F}_{s, t}^{\eta}=\sigma\{\eta(r)-\eta(s) ; s \leq r \leq$ t\} $\vee \mathcal{N}, \mathscr{F}_{t}^{\eta}=\mathscr{F}_{0, t}^{\eta}$.

Let $\left(\Omega^{2}, \mathscr{F}^{2}, P^{2}\right)=(\Omega \times \Omega, \mathscr{F} \otimes \mathscr{F}, P \otimes P)$ be the completion of the product probability space of the above $(\Omega, \mathscr{F}, P)$ with itself, where we define $\mathscr{F}_{t}^{2}=\mathscr{F}_{t} \otimes \mathscr{F}_{t}$ with $t \in[0, T]$ and $\mathscr{F}_{t} \otimes \mathscr{F}_{t}$ being the completion of $\mathscr{F}_{t} \times \mathscr{F}_{t}$. It is worthy of noting that any random variable $\xi=\xi(\omega)$ defined on $\Omega$ can be extended naturally to $\Omega^{2}$ as $\xi^{\prime}\left(\omega, \omega^{\prime}\right)=\xi(\omega)$ with $\left(\omega, \omega^{\prime}\right) \epsilon$ $\Omega^{2}$. For $H=\mathbb{R}^{n}$ and so on, let $L^{1}\left(\Omega^{2}, \mathscr{F}^{2}, P^{2} ; H\right)$ be the set of random variable $\xi: \Omega^{2} \rightarrow H$ which is $\mathscr{F}^{2}$-measurable such that $\mathbb{E}^{2}|\xi| \equiv \int_{\Omega^{2}}\left|\xi\left(\omega^{\prime}, \omega\right)\right| P\left(d \omega^{\prime}\right) P(d \omega)<\infty$. For any $\eta \in L^{1}\left(\Omega^{2}, \mathscr{F}^{2}, P^{2} ; H\right)$, we denote

$$
\mathbb{E}^{\prime} \eta(\omega, \cdot) \doteq \int_{\Omega} \eta\left(\omega, \omega^{\prime}\right) P\left(d \omega^{\prime}\right) .
$$

Particularly, for example, if $\eta_{1}\left(\omega, \omega^{\prime}\right)=\eta_{1}\left(\omega^{\prime}\right)$, then

$$
\mathbb{E}^{\prime} \eta_{1}=\int_{\Omega} \eta_{1}\left(\omega^{\prime}\right) P\left(d \omega^{\prime}\right)=\mathbb{E} \eta_{1} .
$$

We introduce the following notations:

$$
\begin{aligned}
M^{2}\left(0, T ; \mathbb{R}^{n}\right) & \\
= & \left\{v(t, \omega): v(t, \omega) \text { is an } \mathbb{R}^{n}\right. \text {-valued, } \\
& \mathscr{F}_{t} \text {-measurable process }
\end{aligned}
$$

such that $\left.\mathbb{E} \int_{0}^{T}|v(t, \omega)|^{2} d t<\infty\right\}$,

$$
\begin{aligned}
F_{N}^{2} & \left(0, T ; \mathbb{R}^{n}\right) \\
& =\left\{r(t, e, \omega): r(t, e, \omega) \text { is an } \mathbb{R}^{n}\right. \text {-valued, }
\end{aligned}
$$

$\mathscr{F}_{t}$-measurable process

such that $\left.\mathbb{E} \int_{0}^{T} \int_{\mathrm{E}}|r(t, e, \omega)|^{2} \pi(d e) d t<\infty\right\}$,

$$
\begin{aligned}
L_{\pi(\cdot)}^{2} & \left(\mathbb{R}^{n}\right) \\
& =\left\{r(e): r(e) \text { is an } \mathbb{R}^{n}\right. \text {-valued, }
\end{aligned}
$$$$
\mathscr{B}(\text { E) -measurable function }
$$

$$
\begin{aligned}
& \text { such that } \left.\|r\|=\left(\int_{\mathbf{E}}|r(e)|^{2} \pi(d e)\right)^{1 / 2}<\infty\right\}, \\
& L^{2}\left(\Omega, \mathscr{F}_{T}, P ; \mathbb{R}^{n}\right) \\
& =\left\{\xi: \xi \text { is an } \mathbb{R}^{n}\right. \text {-valued, } \\
& \mathscr{F}_{T} \text {-measurable random variable } \\
& \text { such that } \left.\mathbb{E}|\xi|^{2}<\infty\right\} .
\end{aligned}
$$

We use the usual inner product $\langle\cdot, \cdot\rangle$ and Euclidean norm $|\cdot|$ in $\mathbb{R}^{n}, \mathbb{R}^{n \times d}$, and $\mathbb{R}^{n \times l}$. The notation “ $T$ " appearing in the superscripts denotes the transpose of a matrix. All the equalities and inequalities mentioned in this paper are in the sense of $d t \times d P$ almost surely on $[0, T] \times \Omega$.

This work is interested in a class of partial information nonzero sum differential games of MF-BSDEJ, which is inspirited by some interesting financial phenomena. For simplicity, we only consider the case of two players, which is similar for $n$ players. Let us now give a detailed formulation of the problem. Consider the following MF-BSDEJ:

$$
\begin{gathered}
-d y^{v}(t)=\mathbb{E}^{\prime} f\left(t, y^{v}(t), z^{v}(t), r^{v}(t, \cdot),\left(y^{v}(t)\right)^{\prime},\right. \\
\left.\left(z^{v}(t)\right)^{\prime},\left(r^{v}(t, \cdot)\right)^{\prime}, v(t)\right) d t \\
-z^{v}(t) d W(t)-\int_{\mathrm{E}} r^{v}(t, e) \widetilde{N}(d e d t), \\
y^{v}(T)=\xi,
\end{gathered}
$$

where $\xi \in L^{2}\left(\Omega, \mathscr{F}_{T}, P ; \mathbb{R}^{n}\right), f:[0, T] \times \mathbb{R}^{n} \times \mathbb{R}^{n \times d} \times$ $L_{\pi(\cdot)}^{2}\left(\mathbb{R}^{n}\right) \times \mathbb{R}^{n} \times \mathbb{R}^{n \times d} \times L_{\pi(\cdot)}^{2}\left(\mathbb{R}^{n}\right) \times \mathbb{R}^{k_{1}} \times \mathbb{R}^{k_{2}} \rightarrow \mathbb{R}^{n}, v_{1}(\cdot)$ and $v_{2}(\cdot)$ are the control processes of Player 1 and Player 2 , and $v(\cdot)=\left(v_{1}(\cdot), v_{2}(\cdot)\right)$. We always use the subscript 1 (resp., the subscript 2) to characterize the variables corresponding to Player 1 (resp., Player 2). The mean-field backward game system (4) has the meaning that the two players work together to achieve a goal $\xi$ at the terminal time $T$. 
To study our problem, we give some assumptions on $v_{1}(\cdot)$, $v_{2}(\cdot)$, and $f$. Let $U_{i}$ be a nonempty convex subset of $\mathbb{R}^{k_{i}}(i=$ $1,2)$ and $\mathscr{E}_{t}^{i} \subseteq \mathscr{F}_{t}(i=1,2)$ a given subfiltration which represents the information available to Player $i$ at time $t \epsilon$ $[0, T]$, respectively. Now we introduce the admissible control set

$$
\begin{gathered}
\mathscr{U}_{i}=\left\{v_{i}:[0, T] \times \Omega \longrightarrow U_{i} \mid v_{i} \text { is } \mathscr{E}_{t}^{i}\right. \text {-adapted, } \\
\left.\mathbb{E} \int_{0}^{T}\left|v_{i}(t)\right|^{2} d t<\infty\right\}, \quad i=1,2 .
\end{gathered}
$$

Each element of $\mathscr{U}_{i}$ is called an open-loop admissible control for Player $i(i=1,2)$. And $\mathscr{U}_{1} \times \mathscr{U}_{2}$ is called the set of openloop admissible controls for the players.

We assume that

(H1) $f$ is continuously differentiable with respect to $\left(y, z, r, y^{\prime}, z^{\prime}, r^{\prime}, v_{1}, v_{2}\right)$. Moreover, the norm of $f_{y}, f_{z}$, $f_{r}, f_{y^{\prime}}, f_{z^{\prime}}, f_{r^{\prime}}, f_{v_{1}}, f_{v_{2}}$ is bounded by $c>0$.

Now, if both $v_{1}(\cdot)$ and $v_{2}(\cdot)$ are admissible controls and assumption (H1) holds, then MF-BSDEJ (4) admits a unique solution $\left(y^{v_{1}, v_{2}}(\cdot), z^{v_{1}, v_{2}}(\cdot), r^{v_{1}, v_{2}}(\cdot, \cdot)\right) \in M^{2}\left(0, T ; \mathbb{R}^{n}\right) \times$ $M^{2}\left(0, T ; \mathbb{R}^{n \times d}\right) \times F_{N}^{2}\left(0, T ; \mathbb{R}^{n}\right)$ (see Shen and Siu [23]). Ensuring to achieve the goal $\xi$, the players have their own benefits, which are described by the following cost functionals:

$$
\begin{gathered}
J_{i}(v(\cdot)) \\
=\mathbb{E}\left[\int _ { 0 } ^ { T } \mathbb { E } ^ { \prime } l _ { i } \left(t, y^{v}(t), z^{v}(t), r^{v}(t, \cdot),\left(y^{v}(t)\right)^{\prime},\left(z^{v}(t)\right)^{\prime},\right.\right. \\
\left.\left.\left(r^{v}(t, \cdot)\right)^{\prime}, v(t)\right) d t+\Phi_{i}\left(y^{v}(0)\right)\right]
\end{gathered}
$$

where $v(\cdot)=\left(v_{1}(\cdot), v_{2}(\cdot)\right), l_{i}:[0, T] \times \mathbb{R}^{n} \times \mathbb{R}^{n \times d} \times L_{\pi(\cdot)}^{2}\left(\mathbb{R}^{n}\right) \times$ $\mathbb{R}^{n} \times \mathbb{R}^{n \times d} \times L_{\pi(\cdot)}^{2}\left(\mathbb{R}^{n}\right) \times \mathbb{R}^{k_{1}} \times \mathbb{R}^{k_{2}} \rightarrow \mathbb{R}, \Phi_{i}: \mathbb{R}^{n} \rightarrow \mathbb{R}, \quad i=1,2$, satisfying the condition

$$
\begin{gathered}
\mathbb{E}\left[\int_{0}^{T} \mid \mathbb{E}^{\prime} l_{i}\left(t, y^{v}(t), z^{v}(t), r^{v}(t, \cdot),\left(y^{v}(t)\right)^{\prime},\right.\right. \\
\left.\left(z^{v}(t)\right)^{\prime},\left(r^{v}(t, \cdot)\right)^{\prime}, v(t)\right) \mid d t \\
\left.+\left|\Phi_{i}\left(y^{v}(0)\right)\right|\right]<\infty, \quad i=1,2 .
\end{gathered}
$$

We also assume that

(H2) $l_{i}$ is continuously differentiable in $\left(y, z, r, y^{\prime}, z^{\prime}, r^{\prime}\right.$, $\left.v_{1}, v_{2}\right)$ and its partial derivatives are continuous in $\left(y, z, r, y^{\prime}, z^{\prime}, r^{\prime}, v_{1}, v_{2}\right)$ and bounded by $c(1+|y|+$ $\left.|z|+\|r\|+\left|y^{\prime}\right|+\left|z^{\prime}\right|+\left\|r^{\prime}\right\|+\left|v_{1}\right|+\left|v_{2}\right|\right)$. Moreover, $\Phi_{i}$ is continuously differentiable and $\Phi_{i y}$ is bounded by $c(1+|y|)$.

Suppose each player hopes to minimize her/his cost functional $J_{i}\left(v_{1}(\cdot), v_{2}(\cdot)\right)$ by selecting an appropriate admissible control $v_{i}(\cdot)(i=1,2)$. Then the problem is to find a pair of admissible controls $\left(u_{1}(\cdot), u_{2}(\cdot)\right) \in \mathscr{U}_{1} \times \mathscr{U}_{2}$ such that

$$
\begin{aligned}
& J_{1}\left(u_{1}(\cdot), u_{2}(\cdot)\right)=\min _{v_{1}(\cdot) \in \mathscr{U}_{1}} J_{1}\left(v_{1}(\cdot), u_{2}(\cdot)\right), \\
& J_{2}\left(u_{1}(\cdot), u_{2}(\cdot)\right)=\min _{v_{2}(\cdot) \in \mathscr{U}_{2}} J_{2}\left(u_{1}(\cdot), v_{2}(\cdot)\right) .
\end{aligned}
$$

We call the problem above a backward nonzero sum stochastic differential game, where the word backward means that the game system is described by a MF-BSDEJ. For simplicity, we denote it by Problem BNZ. If we can find an admissible control $u(\cdot)=\left(u_{1}(\cdot), u_{2}(\cdot)\right)$ satisfying (8), then we call it an equilibrium point of Problem BNZ and denote the corresponding state trajectory by $(y(\cdot), z(\cdot), r(\cdot, \cdot))=$ $\left(y^{u}(\cdot), z^{u}(\cdot), r^{u}(\cdot, \cdot)\right)$.

\section{A Partial Information Necessary Maximum Principle}

For the convex admissible control set, the classical way to derive necessary optimality conditions is to use the convex perturbation method. Let $u(\cdot)=\left(u_{1}(\cdot), u_{2}(\cdot)\right)$ be an equilibrium point of Problem BNZ and let $(y(\cdot), z(\cdot), r(\cdot, \cdot))$ be the corresponding optimal trajectory. Let $\left(v_{1}(\cdot), v_{2}(\cdot)\right)$ be such that $\left(u_{1}(\cdot)+v_{1}(\cdot), u_{2}(\cdot)+v_{2}(\cdot)\right) \in \mathscr{u}_{1} \times \mathscr{U}_{2}$. Since $\boldsymbol{U}_{1}$ and $\mathscr{u}_{2}$ are convex, for any $0 \leq \rho \leq 1,\left(u_{1}^{\rho}(\cdot), u_{2}^{\rho}(\cdot)\right)=$ $\left(u_{1}(\cdot)+\rho v_{1}(\cdot), u_{1}(\cdot)+\rho v_{1}(\cdot)\right)$ is also in $\mathscr{u}_{1} \times \mathscr{u}_{2}$. As illustrated before, we denote by $\left(y^{u_{1}^{\rho}}(\cdot), z^{u_{1}^{\rho}}(\cdot), r^{u_{1}^{\rho}}(\cdot, \cdot)\right)$ and $\left(y^{u_{2}^{\rho}}(\cdot), z^{u_{2}^{\rho}}(\cdot), r^{u_{2}^{\rho}}(\cdot, \cdot)\right)$ the corresponding state trajectories of game system (4) along with the controls $\left(u_{1}^{\rho}(\cdot), u_{2}(\cdot)\right)$ and $\left(u_{1}(\cdot), u_{2}^{\rho}(\cdot)\right)$.

For convenience, we introduce the notations

$$
\begin{gathered}
\varphi(t, \cdot)=\varphi\left(t, y(t), z(t), r(t, \cdot), y^{\prime}(t), z^{\prime}(t),\right. \\
\left.r^{\prime}(t, \cdot), u_{1}(t), u_{2}(t)\right), \\
\varphi^{v}(t, \cdot)=\varphi\left(t, y(t), z(t), r(t, \cdot), y^{\prime}(t), z^{\prime}(t),\right. \\
\left.r^{\prime}(t, \cdot), v_{1}(t), v_{2}(t)\right), \\
\varphi^{u_{1}^{\rho}}(t, \cdot)=\varphi\left(t, y(t), z(t), r(t, \cdot), y^{\prime}(t), z^{\prime}(t),\right. \\
\left.r^{\prime}(t, \cdot), u_{1}^{\rho}(t), u_{2}(t)\right), \\
\varphi^{u_{2}^{\rho}}(t, \cdot)=\varphi\left(t, y(t), z(t), r(t, \cdot), y^{\prime}(t), z^{\prime}(t),\right. \\
\left.r^{\prime}(t, \cdot), u_{1}(t), u_{2}^{\rho}(t)\right),
\end{gathered}
$$

where $\varphi$ denotes one of $f$ and $l$. 
We introduce the variational equations as follows:

$$
\begin{aligned}
& -d y_{i}^{1}(t) \\
& =\mathbb{E}^{\prime}\left[f_{y}(t, \cdot) y_{i}^{1}(t)+f_{z}(t, \cdot) z_{i}^{1}(t)\right. \\
& \quad+\int_{\mathrm{E}} f_{r}(t, e) r_{i}^{1}(t, e) \pi(d e) \\
& \quad+f_{y^{\prime}}(t, \cdot)\left(y_{i}^{1}(t)\right)^{\prime}+f_{z^{\prime}}(t, \cdot)\left(z_{i}^{1}(t)\right)^{\prime} \\
& \left.\quad+\int_{\mathrm{E}} f_{r^{\prime}}(t, e)\left(r_{i}^{1}(t, e)\right)^{\prime} \pi(d e)+f_{v_{i}}(t, \cdot) v_{i}(t)\right] d t \\
& -z_{i}^{1}(t) d W(t)-\int_{\mathrm{E}} r_{i}^{1}(t, e) \widetilde{N}(d e d t), \\
& y_{i}^{1}(T)=0, \quad(i=1,2) .
\end{aligned}
$$

By (H1), it is easy to know that (10) admits unique adapted solution $\left(y_{i}^{1}(t), z_{i}^{1}(t), r_{i}^{1}(t, \cdot)\right) \in M^{2}\left(0, T ; \mathbb{R}^{n}\right) \times$ $M^{2}\left(0, T ; \mathbb{R}^{n \times d}\right) \times F_{N}^{2}\left(0, T ; \mathbb{R}^{n}\right), i=1,2$.

For $t \in[0, T], \rho>0$, we set

$$
\begin{aligned}
\tilde{y}_{i}^{\rho}(t) & =\frac{y^{u_{i}^{\rho}}(t)-y(t)}{\rho}-y_{i}^{1}(t), \\
\tilde{z}_{i}^{\rho}(t) & =\frac{z^{u_{i}^{\rho}}(t)-z(t)}{\rho}-z_{i}^{1}(t), \\
\tilde{r}_{i}^{\rho}(t, \cdot) & =\frac{r^{u_{i}^{\rho}}(t, \cdot)-r(t, \cdot)}{\rho}-r_{i}^{1}(t, \cdot), \quad(i=1,2) .
\end{aligned}
$$

We have the following.

Lemma 1. Let assumptions (H1) and (H2) hold. Then, for $i=$ 1,2 ,

$$
\begin{aligned}
& \lim _{\rho \rightarrow 0} \sup _{0 \leq t \leq T} \mathbb{E}\left|\tilde{y}_{i}^{\rho}(t)\right|^{2}=0, \\
& \lim _{\rho \rightarrow 0} \mathbb{E} \int_{0}^{T}\left|\widetilde{z}_{i}^{\rho}(t)\right|^{2} d t=0, \\
& \lim _{\rho \rightarrow 0} \mathbb{E} \int_{0}^{T}\left\|\widetilde{r}_{i}^{\rho}(t)\right\|^{2} d t=0 .
\end{aligned}
$$

Proof. For $i=1$, we have

$$
\begin{aligned}
& -d \widetilde{y}_{1}^{\rho}(t) \\
& =\left[\frac{1}{\rho} \mathbb{E}^{\prime}\left(f^{u_{1}^{\rho}}(t, \cdot)-f(t, \cdot)\right)\right. \\
& \quad-\mathbb{E}^{\prime}\left(f_{y}(t, \cdot) y_{1}^{1}(t)+f_{z}(t, \cdot) z_{1}^{1}(t)\right. \\
& \quad+\int_{\mathbb{E}} f_{r}(t, e) r_{1}^{1}(t, e) \pi(d e)+f_{y^{\prime}}(t, \cdot)\left(y_{1}^{1}(t)\right)^{\prime} \\
& \quad+f_{z^{\prime}}(t, \cdot)\left(z_{1}^{1}(t)\right)^{\prime}
\end{aligned}
$$

$$
\begin{gathered}
+\int_{\mathrm{E}} f_{r^{\prime}}(t, e)\left(r_{1}^{1}(t, e)\right)^{\prime} \pi(d e) \\
\left.\left.+f_{v_{1}}(t, \cdot) v_{1}(t)\right)\right] d t \\
-\widetilde{z}_{1}^{\rho}(t) d W(t)-\int_{\mathbf{E}} \widetilde{r}_{1}^{\rho}(t, e) \widetilde{N}(d e d t), \\
\widetilde{y}_{1}^{\rho}(T)=0
\end{gathered}
$$

or

$$
\begin{aligned}
& -d \tilde{y}_{1}^{\rho}(t) \\
& =\mathbb{E}^{\prime}\left[A_{1}^{\rho}(t, \cdot) \tilde{y}_{1}^{\rho}(t)+B_{1}^{\rho}(t, \cdot) \widetilde{z}_{1}^{\rho}(t)\right. \\
& \quad+\int_{\mathrm{E}} C_{1}^{\rho}(t, e) \widetilde{r}_{1}^{\rho}(t, e) \pi(d e) \\
& \quad+D_{1}^{\rho}(t, \cdot)\left(\widetilde{y}_{1}^{\rho}(t)\right)^{\prime}+E_{1}^{\rho}(t, \cdot)\left(\widetilde{z}_{1}^{\rho}(t)\right)^{\prime} \\
& \left.\quad+\int_{\mathrm{E}} F_{1}^{\rho}(t, e)\left(\widetilde{r}_{1}^{\rho}(t, e)\right)^{\prime} \pi(d e)+G_{1}^{\rho}(t, \cdot)\right] d t \\
& -\widetilde{z}_{1}^{\rho}(t) d W(t)-\int_{\mathrm{E}} \widetilde{r}_{1}^{\rho}(t, e) \widetilde{N}(d e d t), \\
& \tilde{y}_{1}^{\rho}(T)=0,
\end{aligned}
$$

where we denote

$(\Theta)$

$$
\begin{aligned}
= & \left(t, y(t)+\lambda \rho\left(y_{1}^{1}(t)+\tilde{y}_{1}^{\rho}(t)\right), z(t)\right. \\
& +\lambda \rho\left(z_{1}^{1}(t)+\widetilde{z}_{1}^{\rho}(t)\right), r(t, \cdot) \\
& +\lambda \rho\left(r_{1}^{1}(t, \cdot)+\widetilde{r}_{1}^{\rho}(t, \cdot)\right),(y(t))^{\prime} \\
& +\lambda \rho\left(\left(y_{1}^{1}(t)\right)^{\prime}+\left(\widetilde{y}_{1}^{\rho}(t)\right)^{\prime}\right),(z(t))^{\prime} \\
& +\lambda \rho\left(\left(z_{1}^{1}(t)\right)^{\prime}+\left(\widetilde{z}_{1}^{\rho}(t)\right)^{\prime}\right),(r(t, \cdot))^{\prime} \\
& +\lambda \rho\left(\left(r_{1}^{1}(t, \cdot)\right)^{\prime}+\left(\widetilde{r}_{1}^{\rho}(t, \cdot)\right)^{\prime}\right), u_{1}(t) \\
& \left.+\lambda \rho v_{1}(t), u_{2}(t)\right), \\
A_{1}^{\rho}(t, \cdot)= & \int_{0}^{1} f_{y}(\Theta) d \lambda, \quad B_{1}^{\rho}(t, \cdot)=\int_{0}^{1} f_{z}(\Theta) d \lambda \\
C_{1}^{\rho}(t, \cdot)= & \int_{0}^{1} f_{r}(\Theta) d \lambda, \quad D_{1}^{\rho}(t, \cdot)=\int_{0}^{1} f_{y^{\prime}}(\Theta) d \lambda \\
E_{1}^{\rho}(t, \cdot)= & \int_{0}^{1} f_{z^{\prime}}(\Theta) d \lambda, \quad F_{1}^{\rho}(t, \cdot)=\int_{0}^{1} f_{r^{\prime}}(\Theta) d \lambda \\
G_{1}^{\rho}(t, \cdot) &
\end{aligned}
$$




$$
\begin{aligned}
& +\left[A_{1}^{\rho}(t, \cdot)-f_{y}(t, \cdot)\right] y_{1}^{1}(t)+\left[B_{1}^{\rho}(t, \cdot)-f_{z}(t, \cdot)\right] z_{1}^{1}(t) \\
& +\left[C_{1}^{\rho}(t, \cdot)-f_{r}(t, \cdot)\right] r_{1}^{1}(t, \cdot) \\
& +\left[D_{1}^{\rho}(t, \cdot)-f_{y^{\prime}}(t, \cdot)\right]\left(y_{1}^{1}(t)\right)^{\prime} \\
& +\left[E_{1}^{\rho}(t, \cdot)-f_{z^{\prime}}(t, \cdot)\right]\left(z_{1}^{1}(t)\right)^{\prime} \\
& +\left[F_{1}^{\rho}(t, \cdot)-f_{r^{\prime}}(t, \cdot)\right]\left(r_{1}^{1}(t, \cdot)\right)^{\prime} .
\end{aligned}
$$

Applying Itô's formula to $\left|\tilde{y}_{1}^{\rho}(t)\right|^{2}$ on $[t, T]$, by virtue of $(\mathrm{H} 1)$, we get

$$
\begin{gathered}
\mathbb{E}\left|\tilde{y}_{1}^{\rho}(t)\right|^{2}+\mathbb{E} \int_{t}^{T}\left(\left|\tilde{z}_{1}^{\rho}(s)\right|^{2}+\left\|\widetilde{r}_{1}^{\rho}(s)\right\|^{2}\right) d s \\
=2 \mathbb{E} \mathbb{E}^{\prime} \int_{t}^{T} \int_{\mathbf{E}} \mid\left\langle\tilde{y}_{1}^{\rho}(s), A_{1}^{\rho}(s, \cdot) \tilde{y}_{1}^{\rho}(s)+B_{1}^{\rho}(s, \cdot) \widetilde{z}_{1}^{\rho}(s)\right. \\
+\int_{\mathbb{E}} C_{1}^{\rho}(s, e) \widetilde{r}_{1}^{\rho}(s, e) \pi(d e) \\
+D_{1}^{\rho}(s, \cdot)\left(\tilde{y}_{1}^{\rho}(s)\right)^{\prime}+E_{1}^{\rho}(s, \cdot)\left(\widetilde{z}_{1}^{\rho}(s)\right)^{\prime} \\
+\int_{\mathbb{E}} F_{1}^{\rho}(s, e)\left(\widetilde{r}_{1}^{\rho}(s, e)\right)^{\prime} \pi(d e) \\
\left.+G_{1}^{\rho}(s, \cdot)\right\rangle \mid \pi(d e) d s \\
\leq C_{0} \mathbb{E} \int_{t}^{T}\left|\tilde{y}_{1}^{\rho}(s)\right|^{2} d s \\
+\frac{1}{2} \mathbb{E} \int_{t}^{T}\left(\left|\tilde{z}_{1}^{\rho}(s)\right|^{2}+\left\|\tilde{r}_{1}^{\rho}(s)\right\|^{2}\right) d s \\
+C_{1} \alpha\left(\mathbb{E} \int_{t}^{T}\left|G_{1}^{\rho}(s)\right|^{2}\right) d s .
\end{gathered}
$$

By Gronwall's inequality, we easily obtain the desired result. Similarly, we can show that the conclusion holds for $i=2$.

Since $\left(u_{1}(\cdot), u_{2}(\cdot)\right)$ is an equilibrium point of Problem $\mathrm{BNZ}$, then

$$
\begin{aligned}
& \rho^{-1}\left[J_{1}\left(u_{1}^{\rho}(\cdot), u_{2}(\cdot)\right)-J_{1}\left(u_{1}(\cdot), u_{2}(\cdot)\right)\right] \geq 0, \\
& \rho^{-1}\left[J_{2}\left(u_{1}(\cdot), u_{2}^{\rho}(\cdot)\right)-J_{2}\left(u_{1}(\cdot), u_{2}(\cdot)\right)\right] \geq 0 .
\end{aligned}
$$

From this and Lemma 1, we have the following variational inequality.
Lemma 2. Let assumption (H1) hold. Then,

$$
\begin{aligned}
\mathbb{E} \int_{0}^{T} \mathbb{E}^{\prime} & {\left[l_{i y}(t, \cdot) y_{i}^{1}(t)+l_{i z}(t, \cdot) z_{i}^{1}(t)\right.} \\
& +\int_{\mathbb{E}} l_{i r}(t) r_{i}^{1}(t, e) \pi(d e)+l_{i y^{\prime}}(t, \cdot)\left(y_{i}^{1}(t)\right)^{\prime} \\
& +l_{i z^{\prime}}(t, \cdot)\left(z_{i}^{1}(t)\right)^{\prime}+\int_{\mathrm{E}} l_{i r^{\prime}}(t)\left(r_{i}^{1}(t, e)\right)^{\prime} \pi(d e) \\
& \left.+l_{i v_{i}}(t, \cdot) v_{i}(t)\right] d t \\
+ & \mathbb{E}\left[\Phi_{i y}(y(0)) y_{i}^{1}(0)\right] \geq 0, \quad(i=1,2) .
\end{aligned}
$$

Proof. For $i=1$, from (12), we derive

$$
\begin{aligned}
& \rho^{-1} {\left[\Phi_{1}\left(y^{u_{1}^{\rho}}(0)\right)-\Phi_{1}(y(0))\right] } \\
&=\rho^{-1} \mathbb{E} \int_{0}^{1} \Phi_{1 y}\left(y(0)+\lambda\left(y^{u_{1}^{\rho}}(0)-y(0)\right)\right) \\
& \quad \times\left(y^{u_{1}^{\rho}}(0)-y(0)\right) d \lambda \\
& \longrightarrow \mathbb{E}\left[\Phi_{1 y}(y(0)) y_{1}^{1}(0)\right], \quad \rho \longrightarrow 0 .
\end{aligned}
$$

Similarly, we have

$$
\begin{gathered}
\rho^{-1}\left\{\mathbb{E} \int_{0}^{T} \mathbb{E}^{\prime}\left[l_{1}^{u_{1}^{\rho}}(t, \cdot)-l_{1}(t, \cdot)\right] d t\right\} \\
\longrightarrow \mathbb{E} \int_{0}^{T} \mathbb{E}^{\prime}\left[l_{1 y}(t, \cdot) y_{1}^{1}(t)+l_{1 z}(t, \cdot) z_{1}^{1}(t)\right. \\
+\int_{\mathbb{E}} l_{1 r}(t) r_{1}^{1}(t, e) \pi(d e)+l_{1 y^{\prime}}(t, \cdot)\left(y_{1}^{1}(t)\right)^{\prime} \\
+l_{1 z^{\prime}}(t, \cdot)\left(z_{1}^{1}(t)\right)^{\prime} \\
+\int_{\mathbb{E}} l_{1 r^{\prime}}(t)\left(r_{1}^{1}(t, e)\right)^{\prime} \pi(d e) \\
\left.+l_{1 v_{1}}(t, \cdot) v_{1}(t)\right] d t \\
\rho \longrightarrow 0 .
\end{gathered}
$$

Let $\rho \rightarrow 0$ in (18); then, it follows that, for $i=1,(20)$ holds. Similarly, we can show that the conclusion holds for $i=2$.

We define the Hamiltonian function $H_{i}:[0, T] \times \mathbb{R}^{n} \times$ $\mathbb{R}^{n \times d} \times L_{\pi(\cdot)}^{2}\left(\mathbb{R}^{n}\right) \times \mathbb{R}^{n} \times \mathbb{R}^{n \times d} \times L_{\pi(\cdot)}^{2}\left(\mathbb{R}^{n}\right) \times \mathbb{R}^{k_{1}} \times \mathbb{R}^{k_{2}} \times \mathbb{R}^{n} \rightarrow \mathbb{R}$, $i=1,2$, as follows:

$$
\begin{gathered}
H_{i}\left(t, y, z, r, y^{\prime}, z^{\prime}, r^{\prime}, v_{1}, v_{2}, p_{i}\right) \\
=-\left\langle f\left(t, y, z, r(\cdot), y^{\prime}, z^{\prime}, r^{\prime}(\cdot), v_{1}, v_{2}\right), p_{i}\right\rangle \\
+l_{i}\left(t, y, z, r(\cdot), y^{\prime}, z^{\prime}, r^{\prime}(\cdot), v_{1}, v_{2}\right), \\
i=1,2 .
\end{gathered}
$$


Let

$$
\begin{aligned}
H_{i}(t, \cdot) & =H_{i}\left(t, y, z, r, y^{\prime}, z^{\prime}, r^{\prime}, u_{1}, u_{2}, p_{i}\right), \\
H_{i}^{v_{1}, v_{2}}(t, \cdot) & =H_{i}\left(t, y, z, r, y^{\prime}, z^{\prime}, r^{\prime}, v_{1}, v_{2}, p_{i}\right), \quad i=1,2 .
\end{aligned}
$$

We introduce the following adjoint equation:

$$
\begin{aligned}
& d p_{i}^{v_{1}, v_{2}}(t) \\
& =-\mathbb{E}^{\prime}\left[H_{i y}^{v_{1}, v_{2}}(t, \cdot)+H_{i y^{\prime}}^{v_{1}, v_{2}}(t, \cdot)\right] d t \\
& -\mathbb{E}^{\prime}\left[H_{i z}^{v_{1}, v_{2}}(t, \cdot)+H_{i z^{\prime}}^{v_{1}, v_{2}}(t, \cdot)\right] d W(t) \\
& -\int_{\mathbb{E}} \mathbb{E}^{\prime}\left[H_{i r}^{v_{1}, v_{2}}(t, e)+H_{i r^{\prime}}^{v_{1}, v_{2}}(t, e)\right] \widetilde{N}(d e d t), \\
& p_{i}^{v_{1}, v_{2}}(0)=-\Phi_{i y}(y(0)), \quad(i=1,2) .
\end{aligned}
$$

Starting from the variational inequality (20), we can now state necessary optimality conditions.

Theorem 3 (partial information necessary maximum principle). Suppose (H1) and (H2) hold. Suppose $\left(u_{1}(\cdot), u_{2}(\cdot)\right)$ is an equilibrium point of Problem $B N Z$ and $(y(\cdot), z(\cdot), r(\cdot, \cdot))$ is the corresponding state trajectory. Then one has that

$$
\begin{aligned}
& \mathbb{E}\left[\left\langle H_{1 v_{1}}(t, \cdot), v_{1}-u_{1}(t)\right\rangle \mid \mathscr{E}_{t}^{1}\right] \geq 0 \\
& \mathbb{E}\left[\left\langle H_{2 v_{2}}(t, \cdot), v_{2}-u_{2}(t)\right\rangle \mid \mathscr{E}_{t}^{2}\right] \geq 0,
\end{aligned}
$$

hold for any $\left(v_{1}, v_{2}\right) \in U_{1} \times U_{2}$, a.e., a.s., where $p_{i}(\cdot)(i=1,2)$ is the solution of the adjoint equation (25).

Proof. For $i=1$, applying Itô's formula to $\left\langle y_{1}^{1}(t), p_{1}(t)\right\rangle$, we obtain

$$
\begin{aligned}
\mathbb{E} \int_{0}^{T} \mathbb{E}^{\prime}[ & l_{1 y}(t, \cdot) y_{1}^{1}(t)+l_{1 z}(t, \cdot) z_{1}^{1}(t) \\
& +\int_{\mathbb{E}} l_{1 r}(t, e) r_{1}^{1}(t, e) \pi(d e)+l_{1 y^{\prime}}(t, \cdot)\left(y_{1}^{1}(t)\right)^{\prime} \\
& +l_{1 z^{\prime}}(t, \cdot)\left(z_{1}^{1}(t)\right)^{\prime}+\int_{\mathbb{E}} l_{1 r^{\prime}}(t, e)\left(r_{1}^{1}(t, e)\right)^{\prime} \pi(d e) \\
& \left.+l_{1 v_{1}}(t) v_{1}(t)\right] d t+\mathbb{E}\left[\Phi_{1 y}(y(0)) y_{1}^{1}(0)\right] \\
=\mathbb{E}\langle & \left.-f_{v_{1}}^{T}(t) p_{1}(t)+l_{1 v_{1}}(t), v_{1}(t)\right\rangle d t .
\end{aligned}
$$

From Lemma 2, it follows that we have

$$
\mathbb{E} \int_{0}^{T}\left\langle H_{1 v_{1}}(t, \cdot), v_{1}(t)\right\rangle d t \geq 0
$$

Because $v_{1}(t)$ satisfies $u_{1}(t)+v_{1}(t) \in \mathscr{U}_{1}$, we have

$$
\mathbb{E} \int_{0}^{T}\left\langle H_{1 v_{1}}(t, \cdot), v_{1}-u_{1}(t)\right\rangle d t \geq 0, \quad \forall v_{1} \in U_{1}
$$

This implies that

$$
\mathbb{E}\left\langle H_{1 v_{1}}(t, \cdot), v_{1}-u_{1}(t)\right\rangle \geq 0, \quad \forall v_{1} \in U_{1} .
$$

Now, let $v_{1}(t) \in U_{1}$ be a deterministic element and let $F$ be an arbitrary element of the $\sigma$-algebra $\mathscr{E}_{t}^{1}$. And set

$$
w_{1}(t)=v_{1}(t) \mathbf{1}_{F}+u_{1}(t) \mathbf{1}_{\Omega-F} .
$$

It is obvious that $w_{1}$ is an admissible control.

Applying the above inequality with $w_{1}$, we get

$$
\mathbb{E}\left[\mathbf{1}_{F}\left\langle H_{1 v_{1}}(t, \cdot), v_{1}-u_{1}(t)\right\rangle\right] \geq 0, \quad \forall F \in \mathscr{E}_{t}^{1},
$$

which implies that

$$
\mathbb{E}\left[\left\langle H_{1 v_{1}}(t, \cdot), v_{1}-u_{1}(t)\right\rangle \mid \mathscr{E}_{t}^{1}\right] \geq 0, \quad \forall v_{1} \in U_{1} \text {, a.e., a.s. }
$$

Proceeding in the same way as the above proof, we can show that the other inequality holds for any $v_{2} \in U_{2}$. Then the proof is completed.

\section{A Partial Information Sufficient Maximum Principle}

In this section, we investigate a sufficient maximum principle for Problem BNZ. Let $\left(y(t), z(t), r(t, \cdot), u_{1}(t), u_{2}(t)\right)$ be a quintuple satisfying (4) and suppose that there exists a solution $p_{i}(t)$ of the corresponding adjoint forward SDE (25). We assume that

(H3) for $i=1,2$, for all $t \in[0, T], H_{i}\left(t, y, z, r, y^{\prime}, z^{\prime}, r^{\prime}\right.$, $\left.v_{1}, v_{2}, p_{i}\right)$ is convex in $\left(y, z, r, y^{\prime}, z^{\prime}, r^{\prime}, v_{1}, v_{2}\right)$, and $\Phi_{i}(y)$ is convex in $y$.

Let

$$
\begin{aligned}
& H_{i}(t, \cdot)=H_{i}\left(t, y(t), z(t), r(t, \cdot), y^{\prime}(t), z^{\prime}(t),\right. \\
& \left.r^{\prime}(t, \cdot), u_{1}(t), u_{2}(t), p_{i}(t)\right), \\
& H_{i}^{v_{1}}(t, \cdot)=H_{i}\left(t, y(t), z(t), r(t, \cdot), y^{\prime}(t), z^{\prime}(t)\right. \text {, } \\
& \left.r^{\prime}(t, \cdot), v_{1}(t), u_{2}(t), p_{i}(t)\right), \\
& H_{i}^{v_{2}}(t, \cdot)=H_{i}\left(t, y(t), z(t), r(t, \cdot), y^{\prime}(t), z^{\prime}(t)\right. \text {, } \\
& \left.r^{\prime}(t, \cdot), u_{1}(t), v_{2}(t), p_{i}(t)\right), \quad i=1,2, \\
& \varphi(t, \cdot)=\varphi\left(t, y(t), z(t), r(t, \cdot), y^{\prime}(t), z^{\prime}(t),\right. \\
& \left.r^{\prime}(t, \cdot), u_{1}(t), u_{2}(t)\right), \\
& \varphi^{v_{1}}(t, \cdot)=\varphi\left(t, y(t), z(t), r(t, \cdot), y^{\prime}(t), z^{\prime}(t),\right. \\
& \left.r^{\prime}(t, \cdot), v_{1}(t), u_{2}(t)\right), \\
& \varphi^{v_{2}}(t, \cdot)=\varphi\left(t, y(t), z(t), r(t, \cdot), y^{\prime}(t), z^{\prime}(t),\right. \\
& \left.r^{\prime}(t, \cdot), u_{1}(t), v_{2}(t)\right),
\end{aligned}
$$

where $\varphi$ denotes one of $f$ and $l$. 
Theorem 4 (partial information sufficient maximum principle). Assume that (H1)-(H3) are satisfied. Moreover, the following partial information maximum conditions hold:

$$
\begin{aligned}
& \mathbb{E}\left[H_{1}(t, \cdot) \mid \mathscr{E}_{t}^{1}\right]=\min _{v_{1} \in \mathscr{U}_{1}} \mathbb{E}\left[H_{1}^{v_{1}}(t, \cdot) \mid \mathscr{E}_{t}^{1}\right], \\
& \mathbb{E}\left[H_{2}(t, \cdot) \mid \mathscr{E}_{t}^{2}\right]=\min _{v_{2} \in \mathscr{U}_{2}} \mathbb{E}\left[H_{2}^{v_{2}}(t, \cdot) \mid \mathscr{E}_{t}^{2}\right] .
\end{aligned}
$$

Then $\left(u_{1}(\cdot), u_{2}(\cdot)\right)$ is an equilibrium point of Problem BNZ.

Proof. For any $v_{1}(\cdot) \in \mathcal{U}_{1}$, we consider

$$
J_{1}\left(v_{1}(\cdot), u_{2}(\cdot)\right)-J_{1}\left(u_{1}(\cdot), u_{2}(\cdot)\right)=\mathbf{I}_{1}+\mathbf{I}_{2},
$$

where

$$
\begin{aligned}
& \mathbf{I}_{1}=\mathbb{E} \int_{0}^{T} \mathbb{E}^{\prime}\left[l_{1}^{v_{1}}(t, \cdot)-l_{1}(t, \cdot)\right] d t, \\
& \mathbf{I}_{2}=\mathbb{E}\left[\Phi_{1}\left(y^{v_{1}}(0)\right)-\Phi_{1}(y(0))\right] .
\end{aligned}
$$

Now applying Itô's formula to $\left\langle p_{1}(t), y^{v_{1}}(t)-y(t)\right\rangle$ on $[0, T]$, we get

$$
\begin{aligned}
& \mathbb{E}\left\langle\Phi_{1 y}(y(0)), y^{v_{1}}(0)-y(0)\right\rangle \\
& =\mathbb{E} \int_{0}^{T} \mathbb{E}^{\prime}\left[\left\langle y^{v_{1}}(t)-y(t),-H_{1 y}(t, \cdot)\right\rangle\right. \\
& \left.+\left\langle\left(y^{v_{1}}(t)\right)^{\prime}-y^{\prime}(t),-H_{1 y^{\prime}}(t, \cdot)\right\rangle\right] d t \\
& +\mathbb{E} \int_{0}^{T} \mathbb{E}^{\prime}\left[\left\langle z^{v_{1}}(t)-z(t),-H_{1 z}(t, \cdot)\right\rangle\right. \\
& \left.+\left\langle\left(z^{v_{1}}(t)\right)^{\prime}-z^{\prime}(t),-H_{1 z^{\prime}}(t, \cdot)\right\rangle\right] d t \\
& +\mathbb{E} \int_{0}^{T} \int_{\mathbb{E}} \mathbb{E}^{\prime}\left[\left\langle r^{v_{1}}(t, e)-r(t, e),-H_{1 r}(t, e)\right\rangle\right. \\
& \quad+\left\langle\left(r^{v_{1}}(t, e)\right)^{\prime}-(r(t, e))^{\prime},\right. \\
& \left.\left.-H_{1 r^{\prime}}(t, e)\right\rangle\right] \pi(d e) d t \\
& -\mathbb{E} \int_{0}^{T} \mathbb{E}^{\prime}\left[\left\langle p_{1}(t), f^{v_{1}}(t, \cdot)-f(t, \cdot)\right\rangle\right] d t .
\end{aligned}
$$

Moreover, by virtue of (39) and convexity of $\Phi_{1}$, it instantly follows that

$$
\mathbf{I}_{2} \geq \mathbb{E}\left\langle\Phi_{1 y}(y(0)), y^{v_{1}}(0)-y(0)\right\rangle=-\Xi_{1}+\Xi_{2},
$$

where

$$
\begin{gathered}
\Xi_{1}=\mathbb{E} \int_{0}^{T} \mathbb{E}^{\prime}\left[\left\langle y^{\nu_{1}}(t)-y(t), H_{1 y}(t, \cdot)\right\rangle\right. \\
\left.+\left\langle\left(y^{v_{1}}(t)\right)^{\prime}-y^{\prime}(t), H_{1 y^{\prime}}(t, \cdot)\right\rangle\right] d t \\
+\mathbb{E} \int_{0}^{T} \mathbb{E}^{\prime}\left[\left\langle z^{\nu_{1}}(t)-z(t), H_{1 z}(t, \cdot)\right\rangle\right. \\
\left.+\left\langle\left(z^{v_{1}}(t)\right)^{\prime}-z^{\prime}(t), H_{1 z^{\prime}}(t, \cdot)\right\rangle\right] d t \\
+\mathbb{E} \int_{0}^{T} \int_{\mathbb{E}} \mathbb{E}^{\prime}\left[\left\langle r^{v_{1}}(t, e)-r(t, e), H_{1 r}(t, e)\right\rangle\right. \\
\left.\quad+\left\langle\left(r^{\nu_{1}}(t, e)\right)^{\prime}-(r(t, e))^{\prime}, H_{1 r^{\prime}}(t, e)\right\rangle\right] \\
\times \pi(d e) d t, \\
\Xi_{2}=-\mathbb{E} \int_{0}^{T} \mathbb{E}^{\prime}\left[\left\langle p_{1}(t), f^{v_{1}}(t, \cdot)-f(t, \cdot)\right\rangle\right] d t .
\end{gathered}
$$

Noting the definition of $H_{1}$ and $\mathbf{I}_{1}$, we have

$$
\begin{aligned}
\mathbf{I}_{1}= & \mathbb{E} \int_{0}^{T} \mathbb{E}^{\prime}\left[H_{1}^{v_{1}}(t, \cdot)-H_{1}(t, \cdot)\right] d t \\
& +\mathbb{E} \int_{0}^{T} \mathbb{E}^{\prime}\left[\left\langle p_{1}(t), f^{v_{1}}(t, \cdot)-f(t, \cdot)\right\rangle\right] d t \\
= & \Xi_{3}-\Xi_{2},
\end{aligned}
$$

where

$$
\Xi_{3}=\mathbb{E} \int_{0}^{T} \mathbb{E}^{\prime}\left[H_{1}^{v_{1}}(t, \cdot)-H_{1}(t, \cdot)\right] d t .
$$

Using convexity of $H_{1}\left(t, y, z, r, y^{\prime}, z^{\prime}, r^{\prime}, v_{1}, v_{2}, p_{1}\right)$ with respect to $\left(y, z, r, y^{\prime}, z^{\prime}, r^{\prime}, v_{1}, v_{2}\right)$, we obtain

$$
\begin{aligned}
& H_{1}^{v_{1}}(t, \cdot)-H_{1}(t, \cdot) \\
& \geq H_{1 y}(t)\left(y^{v_{1}}(t)-y(t)\right)+H_{1 z}(t, \cdot)\left(z^{v_{1}}(t)-z(t)\right) \\
& \quad+\int_{\mathbf{E}} H_{1 r}(t, e)\left(r^{v_{1}}(t, e)-r(t, e)\right) \pi(d e) \\
& \quad+H_{1 y^{\prime}}(t, \cdot)\left(\left(y^{v_{1}}(t)\right)^{\prime}-y^{\prime}(t)\right) \\
& \quad+H_{1 z^{\prime}}(t, \cdot)\left(\left(z^{v_{1}}(t)\right)^{\prime}-z^{\prime}(t)\right) \\
& \quad+\int_{\mathbf{E}} H_{1 r^{\prime}}(t, e)\left(\left(r^{v_{1}}(t, e)\right)^{\prime}-r(t, e)\right) \pi(d e) \\
& \quad+H_{1 u_{1}}(t, \cdot)\left(v_{1}(t)-u_{1}(t)\right) .
\end{aligned}
$$

Since $v_{1} \rightarrow \mathbb{E}\left[H_{1}^{v_{1}}(t, \cdot) \mid \mathscr{E}_{t}^{1}\right], v_{1} \in \mathscr{U}_{1}$, is minimal for $u_{1}(t)$ and $v_{1}(t)$ and $u_{1}(t)$ are $\mathscr{E}_{t}^{1}$-measurable, we get

$$
\begin{aligned}
& \mathbb{E}\left[H_{1 u_{1}}(t, \cdot) \mid \mathscr{E}_{t}^{1}\right]\left(v_{1}(t)-u_{1}(t)\right) \\
& \quad=\mathbb{E}\left[H_{1 u_{1}}(t, \cdot)\left(v_{1}(t)-u_{1}(t)\right) \mid \mathscr{E}_{t}^{1}\right] \geq 0 .
\end{aligned}
$$


Hence combining (43), (44), and (45), we obtain

$$
\begin{aligned}
& \Xi_{3} \\
& \geq \mathbb{E} \int_{0}^{T} \mathbb{E}^{\prime}\left[\left\langle y^{v_{1}}(t, \cdot)-y(t),-H_{1 y}(t, \cdot)\right\rangle\right. \\
& \left.+\left\langle\left(y^{v_{1}}(t)\right)^{\prime}-y^{\prime}(t),-H_{1 y^{\prime}}(t, \cdot)\right\rangle\right] d t \\
& +\mathbb{E} \int_{0}^{T} \mathbb{E}^{\prime}\left[\left\langle z^{v_{1}}(t)-z(t),-H_{1 z}(t, \cdot)\right\rangle\right. \\
& \left.+\left\langle\left(z^{v_{1}}(t)\right)^{\prime}-z^{\prime}(t),-H_{1 z^{\prime}}(t, \cdot)\right\rangle\right] d t \\
& +\mathbb{E} \int_{0}^{T} \int_{\mathbb{E}} \mathbb{E}^{\prime}\left[\left\langle r^{v_{1}}(t, e)-r(t, e), H_{1 r}(t, e)\right\rangle\right. \\
& \left.+\left\langle\left(r^{v_{1}}(t, e)\right)^{\prime}-(r(t, e))^{\prime}, H_{1 r^{\prime}}(t, e)\right\rangle\right] \\
& \times \pi(d e) d t=\Xi_{1} \text {. }
\end{aligned}
$$

Therefore, it follows from (35), (40), and (46) that

$$
\begin{aligned}
J_{1} & \left(v_{1}(\cdot), u_{2}(\cdot)\right)-J_{1}\left(u_{1}(\cdot), u_{2}(\cdot)\right) \\
& \geq \Xi_{3}-\Xi_{2}-\Xi_{1}+\Xi_{2} \\
& \geq \Xi_{1}-\Xi_{2}-\Xi_{1}+\Xi_{2}=0 .
\end{aligned}
$$

Then it implies that

$$
J_{1}\left(u_{1}(\cdot), u_{2}(\cdot)\right)=\min _{v_{1}(\cdot) \in \mathscr{U}_{1}} J_{1}\left(v_{1}(\cdot), u_{2}(\cdot)\right) .
$$

In the same way

$$
J_{2}\left(u_{1}(\cdot), u_{2}(\cdot)\right)=\min _{v_{2}(\cdot) \in \mathscr{U}_{2}} J_{2}\left(u_{1}(\cdot), v_{2}(\cdot)\right) .
$$

Hence, we draw the desired conclusion. The proof is completed.

\section{Application in a Partial Information LQ Case}

In this section we work out an example of partial information linear-quadratic differential games of MF-BSDEJ to illustrate the application of the theoretical results. For notational simplification, we assume $n=d=k_{1}=k_{2}=1, U_{1}=U_{2}=\mathbb{R}$, and $\mathscr{E}_{t}^{1}=\mathscr{E}_{t}^{2}=\mathscr{E}_{t} \subseteq \mathscr{F}_{t}$.

Consider the following:

$$
\begin{aligned}
& -d y^{v_{1}, v_{2}}(t) \\
& =\mathbb{E}^{\prime}\left[A(t) y^{v_{1}, v_{2}}(t)+C(t) z^{v_{1}, v_{2}}(t)+D(t) r^{v_{1}, v_{2}}(t, \cdot)\right. \\
& +\bar{A}(t)\left(y^{v_{1}, v_{2}}(t)\right)^{\prime}+\bar{C}(t)\left(z^{v_{1}, v_{2}}(t)\right)^{\prime} \\
& +\bar{D}(t)\left(r^{v_{1}, v_{2}}(t, \cdot)\right)^{\prime}+B_{1}(t) v_{1}(t) \\
& \left.+B_{2}(t) v_{2}(t)\right] d t \\
& -z^{v_{1}, v_{2}}(t) d W(t)-\int_{\mathrm{E}} r^{v_{1}, v_{2}}(t, e) \widetilde{N}(\text { ded } d t), \\
& y^{v_{1}, v_{2}}(T)=\xi .
\end{aligned}
$$

The cost functional is

$$
\begin{aligned}
& J_{i}\left(v_{1}(\cdot), v_{2}(\cdot)\right) \\
& \quad=\frac{1}{2} \mathbb{E}\left[\int _ { 0 } ^ { T } \mathbb { E } ^ { \prime } \left(M_{i}(t) v_{i}^{2}(t)+N_{i}(t)\left(y^{v_{1}, v_{2}}(t)\right)^{2}\right.\right. \\
& \left.+\bar{N}_{i}(t)\left(\left(y^{v_{1}, v_{2}}(t)\right)^{\prime}\right)^{2}\right) d t \\
& \left.+L_{i}\left(y^{v_{1}, v_{2}}(0)\right)^{2}\right], \quad i=1,2
\end{aligned}
$$

where constants $L_{i} \geq 0, i=1,2$. Functions $A(\cdot), \bar{A}(\cdot), B_{1}(\cdot)$, $B_{2}(\cdot), C(\cdot), \bar{C}(\cdot), D(\cdot), \bar{D}(\cdot)$ are bounded and deterministic; $N_{i}(\cdot), \bar{N}_{i}(\cdot), i=1,2$, are nonnegative, bounded, and deterministic; $M_{i}(\cdot), i=1,2$, are positive, bounded, and deterministic; $M_{i}^{-1}(\cdot), i=1,2$, are also bounded. Our task is to find $\left(u_{1}(\cdot), u_{2}(\cdot)\right) \in \mathscr{U}_{1} \times \mathscr{U}_{2}$ such that

$$
\begin{aligned}
& J_{1}\left(u_{1}(\cdot), u_{2}(\cdot)\right)=\min _{v_{1}(\cdot) \in \mathscr{U}_{1}} J_{1}\left(v_{1}(\cdot), u_{2}(\cdot)\right), \\
& J_{2}\left(u_{1}(\cdot), u_{2}(\cdot)\right)=\min _{v_{2}(\cdot) \in \mathscr{U}_{2}} J_{2}\left(u_{1}(\cdot), v_{2}(\cdot)\right) .
\end{aligned}
$$

Theorem 5. The mapping

$$
\begin{aligned}
& u_{1}(t)=M_{1}^{-1}(t) B_{1}(t) \mathbb{E}\left[p_{1}(t) \mid \mathscr{E}_{t}\right], \\
& u_{2}(t)=M_{2}^{-1}(t) B_{2}(t) \mathbb{E}\left[p_{2}(t) \mid \mathscr{E}_{t}\right],
\end{aligned}
$$

is one Nash equilibrium point for the above game problem, where $\left(p_{1}(t), p_{2}(t), y(t), z(t), r(t, \cdot)\right)$ is the solution of the following mean-field forward-backward stochastic differential equations with jumps (MF-FBSDEJ):

$$
d p_{i}(t)
$$

$$
\begin{aligned}
= & \mathbb{E}^{\prime}\left[A(t) p_{i}(t)+\bar{A}(t) p_{i}^{\prime}(t)-N_{i}(t) y(t)\right. \\
& \left.-\bar{N}_{i}(t) y^{\prime}(t)\right] d t \\
+ & \mathbb{E}^{\prime}\left[C(t) p_{i}(t)+\bar{C}(t) p_{i}^{\prime}(t)\right] d W(t) \\
+ & \int_{\mathbb{E}} \mathbb{E}^{\prime}\left[D(t) p_{i}(t)+\bar{D}(t) p_{i}^{\prime}(t)\right] \widetilde{N}(d e d t),
\end{aligned}
$$

$-d y(t)$

$$
\begin{aligned}
=\mathbb{E}^{\prime} & \left\{A(t) y(t)+C(t) z(t)+D(t) r(t, \cdot)+\bar{A}(t) y^{\prime}(t)\right. \\
+ & \bar{C}(t) z^{\prime}(t)+\bar{D}(t) r^{\prime}(t, \cdot)+B_{1}^{2}(t) M_{1}^{-1}(t) \\
\quad & \left.\times \mathbb{E}\left[p_{1}(t) \mid \mathscr{E}_{t}\right]+B_{2}^{2}(t) M_{2}^{-1}(t) \mathbb{E}\left[p_{2}(t) \mid \mathscr{E}_{t}\right]\right\} d t \\
- & z(t) d W(t)-\int_{\mathrm{E}} r(t, e) \widetilde{N}(\text { ded } d t), \\
& p_{i}(0)=-L_{i} y(0), \quad y(T)=\xi, \quad i=1,2 .
\end{aligned}
$$


Proof. We first prove the existence of the solution of (54). We set

$$
\widehat{\theta}(t)=\mathbb{E}\left[\theta(t) \mid \mathscr{E}_{t}\right], \quad \theta=y, z, r, y^{\prime}, z^{\prime}, r^{\prime}, p_{1}, p_{2} .
$$

Similar to Lemma 5.4 of [24], the optimal filter $(\widehat{y}(t), \widehat{z}(t)$, $\left.\widehat{r}(t, \cdot), \widehat{y}^{\prime}(t), \widehat{z}^{\prime}(t), \widehat{r}^{\prime}(t, \cdot), \widehat{p}_{1}(t), \widehat{p}_{2}(t)\right) \quad$ of $\quad(y(t), z(t), r(t, \cdot)$, $\left.y^{\prime}(t), z^{\prime}(t), r^{\prime}(t, \cdot), p_{1}(t), p_{2}(t)\right)$ satisfies

$d \widehat{p}_{i}(t)$

$$
\begin{aligned}
= & \mathbb{E}^{\prime}\left[A(t) \widehat{p}_{i}(t)+\bar{A}(t) \hat{p}_{i}^{\prime}(t)-N_{i}(t) \hat{y}(t)\right. \\
& \left.-\bar{N}_{i}(t) \hat{y}^{\prime}(t)\right] d t \\
+ & \mathbb{E}^{\prime}\left[C(t) \widehat{p}_{i}(t)+\bar{C}(t) \hat{p}_{i}^{\prime}(t)\right] d W(t) \\
+ & \int_{\mathbb{E}} \mathbb{E}^{\prime}\left[D(t) \widehat{p}_{i}(t)+\bar{D}(t) \hat{p}_{i}^{\prime}(t)\right] \widetilde{N}(\text { dedt } d,
\end{aligned}
$$

$-d \widehat{y}(t)$

$$
\begin{aligned}
=\mathbb{E}^{\prime}[A(t) \hat{y}(t)+C(t) \widehat{z}(t)+D(t) \hat{r}(t, \cdot) & \\
& +\bar{A}(t) \widehat{y}^{\prime}(t)+\bar{C}(t) \widehat{z}^{\prime}(t)+\bar{D}(t) \widehat{r}^{\prime}(t, \cdot) \\
& \left.+B_{1}^{2}(t) M_{1}^{-1}(t) \widehat{p}_{1}(t)+B_{2}^{2}(t) M_{2}^{-1}(t) \widehat{p}_{2}(t)\right] d t \\
-\widehat{z}(t) d W(t)-\int_{\mathrm{E}} \widehat{r}(t, e) \widetilde{N}(\text { ded } d), & \widehat{y}(T)=\mathbb{E}\left[\xi \mid \mathscr{E}_{T}\right], \quad i=1,2 .
\end{aligned}
$$

Due to the above analysis, the candidate equilibrium point $\left(u_{1}(\cdot), u_{2}(\cdot)\right)$ can be rewritten as

$$
\begin{aligned}
& u_{1}(t)=M_{1}^{-1}(t) B_{1}(t) \widehat{p}_{1}(t), \\
& u_{2}(t)=M_{2}^{-1}(t) B_{2}(t) \widehat{p}_{2}(t),
\end{aligned}
$$

where $\widehat{p}_{i}(t)(i=1,2)$ admits MF-FBSDEJ (56). We introduce a new MF-FBSDEJ:

$$
\begin{aligned}
& d P(t) \\
& =\mathbb{E}^{\prime}\left[A(t) P(t)+\bar{A}(t) P^{\prime}(t)\right. \\
& \quad-\left(B_{1}^{2}(t) M_{1}^{-1}(t) N_{1}(t)+B_{2}^{2}(t) M_{2}^{-1}(t) N_{2}(t)\right) \\
& \quad \times Y(t)-\left(B_{1}^{2}(t) M_{1}^{-1}(t) \bar{N}_{1}(t)+B_{2}^{2}(t) M_{2}^{-1}(t)\right. \\
& \left.\left.\quad \times \bar{N}_{2}(t)\right) Y^{\prime}(t)\right] d t \\
& +\mathbb{E}^{\prime}\left[C(t) P(t)+\bar{C}(t) P^{\prime}(t)\right] d W(t) \\
& +\int_{\mathbb{E}} \mathbb{E}^{\prime}\left[D(t) P(t)+\bar{D}(t) P^{\prime}(t)\right] \widetilde{N}(\text { de } d t)
\end{aligned}
$$

$$
\begin{aligned}
& -d Y(t) \\
& =\mathbb{E}^{\prime}[A(t) Y(t)+C(t) Z(t)+D(t) R(t, \cdot) \\
& +\bar{A}(t) Y^{\prime}(t)+\bar{C}(t) Z^{\prime}(t)+\bar{D}(t) R^{\prime}(t, \cdot) \\
& +P(t)] d t-Z(t) d W(t) \\
& -\int_{\mathrm{E}} R(t, e) \widetilde{N}(d e d t) \\
& P(0)=-\left[B_{1}^{2}(0) M_{1}^{-1}(0) L_{1}+B_{2}^{2}(0) M_{2}^{-1}(0) L_{2}\right] Y(0), \\
& Y(T)=\xi \text {. }
\end{aligned}
$$

Based on the analysis above, we can say the existence and uniqueness of MF-FBSDEJ (56) are equivalent to those of MF-FBSDEJ (58). It is easy to check that MF-FBSDEJ (58) satisfies assumptions (A3)-(A5) with $H=1, \mu_{1}=1$, and $\mu_{2}=\beta_{2}=0$. According to Theorem 7 in Appendix, there exists a unique solution $(P(t), Y(t), Z(t), R(t, \cdot))$ of $(58)$; here,

$$
\begin{aligned}
& P(t)=B_{1}^{2}(t) M_{1}^{-1}(t) \widehat{p}_{1}(t)+B_{2}^{2}(t) M_{2}^{-1}(t) \widehat{p}_{2}(t), \\
& Y(t)=\widehat{y}(t), \quad Z(t)=\widehat{z}(t), \quad R(t, \cdot)=\widehat{r}(t, \cdot) .
\end{aligned}
$$

Then there exists a unique solution $\left(\widehat{p}_{1}(t), \widehat{p}_{2}(t), \widehat{y}(t), \widehat{z}(t)\right.$, $\widehat{r}(t, \cdot))$ of MF-FBSDEJ (56). Furthermore, there exists at most one equilibrium point for the underlying game.

Now we try to prove that $\left(u_{1}(\cdot), u_{2}(\cdot)\right)$ is a Nash equilibrium point for our backward LQ game problem. We only prove

$$
J_{1}\left(u_{1}(\cdot), u_{2}(\cdot)\right)=\min _{v_{1}(\cdot) \in \mathscr{U}_{1}} J_{1}\left(v_{1}(\cdot), u_{2}(\cdot)\right)
$$

It is similar to getting another inequality of $(52)$. $\left(y^{v_{1}}(t)\right.$, $\left.z^{v_{1}}(t), r^{v_{1}}(t, \cdot)\right)$ denotes the solution of the system

$$
\begin{gathered}
-d y^{v_{1}}(t) \\
=\mathbb{E}^{\prime}\left[A(t) y^{v_{1}}(t)+C(t) z^{v_{1}}(t)+D(t) r^{v_{1}}(t, \cdot)\right. \\
+\bar{A}(t)\left(y^{v_{1}}(t)\right)^{\prime}+\bar{C}(t)\left(z^{v_{1}}(t)\right)^{\prime}+\bar{D}(t)\left(r^{v_{1}}(t, \cdot)\right)^{\prime} \\
\left.+B_{1}(t) v_{1}(t)+B_{2}(t) u_{2}(t)\right] d t \\
-z^{v_{1}}(t) d W(t)-\int_{\mathrm{E}} r^{v_{1}}(t, e) \widetilde{N}(d e d t), \\
y^{v_{1}}(T)=\xi .
\end{gathered}
$$


Then

$$
\begin{aligned}
J_{1}\left(v_{1}(\cdot), u_{2}(\cdot)\right)- & J_{1}\left(u_{1}(\cdot), u_{2}(\cdot)\right) \\
=\frac{1}{2} \mathbb{E}\left[\int_{0}^{T} \mathbb{E}^{\prime}\right. & \left(M_{1}(t)\left(v_{1}(t)-u_{1}(t)\right)^{2}\right. \\
& +2 M_{1}(t) u_{1}(t)\left(v_{1}(t)-u_{1}(t)\right) \\
& +N_{1}(t)\left(y^{v_{1}}(t)-y(t)\right)^{2} \\
& +2 N_{1}(t) y(t)\left(y^{v_{1}}(t)-y(t)\right) \\
& +\bar{N}_{1}(t)\left(\left(y^{v_{1}}(t)\right)^{\prime}-y^{\prime}(t)\right)^{2} \\
& +2 \bar{N}_{1}(t) y^{\prime}(t)\left(\left(y^{v_{1}}(t)\right)^{\prime}-y^{\prime}(t)\right) d t \\
+ & L_{1}\left(y^{v_{1}}(0)-y^{u}(0)\right)^{2} \\
+ & \left.2 L_{1} y^{u}(0)\left(y^{v}(0)-y^{u}(0)\right)\right] .
\end{aligned}
$$

Applying Itô’'s formula to $\left(y^{v_{1}}(t)-y(t)\right) \widehat{p}_{1}(t)$, we have

$$
\begin{aligned}
\mathbb{E}\left\{L_{1} y^{v}(0)\left(y^{v}(0)-y^{u}(0)\right)\right\} & \\
=-\mathbb{E} \int_{0}^{T} \mathbb{E}^{\prime}[ & B_{1}(t) \hat{p}_{1}(t)\left(v_{1}(t)-u_{1}(t)\right) \\
& +N_{1}(t) y(t)\left(y^{v_{1}}(t)-y(t)\right) \\
& \left.+\bar{N}_{1}(t) y^{\prime}(t)\left(\left(y^{v_{1}}(t)\right)^{\prime}-y^{\prime}(t)\right)\right] d t .
\end{aligned}
$$

As $M_{1}(t)>0, N_{1}(t) \geq 0, \bar{N}_{1}(t) \geq 0, \forall t \in[0, T], L_{1} \geq 0$, noting that $u_{1}(t)=M_{1}^{-1}(t) B_{1}(t) \widehat{p}_{1}(t)$, we have

$$
\begin{aligned}
J_{1}\left(v_{1}(\cdot), u_{2}(\cdot)\right)-J_{1}\left(u_{1}(\cdot), u_{2}(\cdot)\right) \\
\geq \mathbb{E} \int_{0}^{T} \mathbb{E}^{\prime}\left[\left(M_{1}(t) u_{1}(t)-B_{1}(t) \widehat{p}_{1}(t)\right)\right. \\
\left.\quad \times\left(v_{1}(t)-u_{1}(t)\right)\right] d t=0 .
\end{aligned}
$$

So $\left(u_{1}(t), u_{2}(t)\right)=\left(M_{1}^{-1}(t) B_{1}(t) \widehat{p}_{1}(t), M_{2}^{-1}(t) B_{2}(t) \widehat{p}_{2}(t)\right)$ is a Nash equilibrium point for our backward LQ nonzero sum differential game problem.

\section{Appendix}

For the sake of convenience and completeness, we cite the existence and uniqueness theorem of MF-BSDEJ obtained by Shen and Siu [23]. They studied the following MF-BSDEJ:

$$
\begin{gathered}
-d y(t) \\
=\mathbb{E}^{\prime} f\left(t, \xi(t), \xi^{\prime}(t), v(t)\right) d t \\
-z(t) d W(t)-\int_{\mathrm{E}} r(t, e) \widetilde{N}(d e d t), \\
y(T)=\xi,
\end{gathered}
$$

where $\left(\xi(t), \xi^{\prime}(t)\right)=\left(y(\cdot), z(\cdot), r(\cdot, \cdot), y^{\prime}(\cdot), z^{\prime}(\cdot), r^{\prime}(\cdot, \cdot)\right) \quad \epsilon$ $\mathbb{R}^{n} \times \mathbb{R}^{n \times d} \times L_{\pi(\cdot)}^{2}\left(\mathbb{R}^{n}\right) \times \mathbb{R}^{n} \times \mathbb{R}^{n \times d} \times L_{\pi(\cdot)}^{2}\left(\mathbb{R}^{n}\right), \xi \in$ $L^{2}\left(\Omega, \mathscr{F}_{T}, P ; \mathbb{R}^{n}\right)$, is a random variable, and $T>0$;

$$
\begin{aligned}
f: & {[0, T] \times \mathbb{R}^{n} \times \mathbb{R}^{n \times d} \times L_{\pi(\cdot)}^{2}\left(\mathbb{R}^{n}\right) } \\
& \times \mathbb{R}^{n} \times \mathbb{R}^{n \times d} \times L_{\pi(\cdot)}^{2}\left(\mathbb{R}^{n}\right) \longrightarrow \mathbb{R}^{n} .
\end{aligned}
$$

They assumed that

(A1) for each $\xi, \xi^{\prime} \in \mathbb{R}^{n} \times \mathbb{R}^{n \times d} \times L_{\pi(\cdot)}^{2}\left(\mathbb{R}^{n}\right), f\left(\cdot, \xi, \xi^{\prime}\right)$ is an $\mathscr{F}_{t}$-measurable process defined on $[0, T]$ with $f(\cdot, 0,0) \in M^{2}\left(0, T ; \mathbb{R}^{n} \times \mathbb{R}^{n \times d}\right) \times F_{N}^{2}\left(0, T ; \mathbb{R}^{n}\right) ;$

(A2) $f\left(t, \zeta, \zeta^{\prime}\right)$ satisfies Lipschitz condition: there exists a constant $l>0$, such that

$$
\begin{gathered}
\left|f\left(t, \xi, \xi^{\prime}\right)-f\left(t, \bar{\xi}^{\prime}, \bar{\xi}^{\prime}\right)\right| \leq l\left(|\xi-\bar{\xi}|+\left|\xi^{\prime}-\bar{\xi}^{\prime}\right|\right), \\
\forall \xi=(y, z, r)^{T}, \quad \xi^{\prime}=\left(y^{\prime}, z^{\prime}, r^{\prime}\right)^{T}, \quad \bar{\xi}=(\bar{y}, \bar{z}, \bar{r})^{T}, \\
\bar{\xi}^{\prime}=\left(\bar{y}^{\prime}, \bar{z}^{\prime}, \bar{r}^{\prime}\right)^{T} \in \mathbb{R}^{n} \times \mathbb{R}^{n \times d} \times L_{\pi(\cdot)}^{2}\left(\mathbb{R}^{n}\right), \quad \forall t \in[0, T] .
\end{gathered}
$$

Based on the fixed-point theorem, Shen and Siu [23] obtained the following existence and uniqueness result.

Proposition 6. One assumes that (A1) and (A2) hold. Then MF-BSDEJ (65) has a unique solution $(y(t), z(t), r(t, \cdot)) \in$ $M^{2}\left(0, T ; \mathbb{R}^{n} \times \mathbb{R}^{n \times d}\right) \times F_{N}^{2}\left(0, T ; \mathbb{R}^{n}\right)$.

In the present paper we research the game problem of a MF-BSDEJ, so the game system and the adjoint equation constitute exactly a kind of initial coupled MF-FBSDEJ. Due to this, we give an existence and uniqueness theorem of MFFBSDEJ. Consider the following MF-FBSDEJ:

$$
\begin{gathered}
d x(t) \\
=\mathbb{E}^{\prime}\left[b \left(t, x(t), y(t), z(t), r(t, \cdot), x^{\prime}(t),\right.\right. \\
\left.\left.y^{\prime}(t), z^{\prime}(t), r^{\prime}(t, \cdot)\right)\right] d t \\
+\mathbb{E}^{\prime}\left[\sigma \left(t, x(t), y(t), z(t), r(t, \cdot), x^{\prime}(t),\right.\right. \\
\left.\left.y^{\prime}(t), z^{\prime}(t), r^{\prime}(t, \cdot)\right)\right] d W(t) \\
+\int_{\mathbb{E}} \mathbb{E}^{\prime}\left[\gamma \left(t, x(t), y(t), z(t), r(t, e), x^{\prime}(t),\right.\right. \\
\left.\left.-d y(t) \quad y^{\prime}(t), z^{\prime}(t), r^{\prime}(t, e), e\right)\right] \widetilde{N}(d e d t), \\
=\mathbb{E}^{\prime}\left[f \left(t, x(t), y(t), r(t, \cdot), x^{\prime}(t),\right.\right. \\
\left.\left.y^{\prime}(t), r^{\prime}(t, \cdot)\right)\right] d t-z(t) d W(t) \\
-\int_{\mathbb{E}} r(t, e) \widetilde{N}(d e d t), \\
x(0)=\psi(y(0)), \quad y(T)=\xi,
\end{gathered}
$$


where $\left(x(\cdot), y(\cdot), z(\cdot), r(\cdot, \cdot), x^{\prime}(\cdot), y^{\prime}(\cdot), z^{\prime}(\cdot), r^{\prime}(\cdot, \cdot)\right) \in \mathbb{R}^{m} \times$ $\mathbb{R}^{n} \times \mathbb{R}^{n \times d} \times L_{\pi(\cdot)}^{2}\left(\mathbb{R}^{n}\right) \times \mathbb{R}^{m} \times \mathbb{R}^{n} \times \mathbb{R}^{n \times d} \times L_{\pi(\cdot)}^{2}\left(\mathbb{R}^{n}\right), \xi \in$ $L^{2}\left(\Omega, \mathscr{F}_{T}, P ; \mathbb{R}^{n}\right)$, is a random variable, and $T>0$;

$$
\begin{aligned}
b: & \Omega \times[0, T] \times \mathbb{R}^{m} \times \mathbb{R}^{n} \times \mathbb{R}^{n \times d} \times L_{\pi(\cdot)}^{2}\left(\mathbb{R}^{n}\right) \\
& \times \mathbb{R}^{m} \times \mathbb{R}^{n} \times \mathbb{R}^{n \times d} \times L_{\pi(\cdot)}^{2}\left(\mathbb{R}^{n}\right) \longrightarrow \mathbb{R}^{m}, \\
\sigma & : \Omega \times[0, T] \times \mathbb{R}^{m} \times \mathbb{R}^{n} \times \mathbb{R}^{n \times d} \times L_{\pi(\cdot)}^{2}\left(\mathbb{R}^{n}\right) \\
& \times \mathbb{R}^{m} \times \mathbb{R}^{n} \times \mathbb{R}^{n \times d} \times L_{\pi(\cdot)}^{2}\left(\mathbb{R}^{n}\right) \longrightarrow \mathbb{R}^{m \times d}, \\
\gamma: & \Omega \times[0, T] \times \mathbb{R}^{m} \times \mathbb{R}^{n} \times \mathbb{R}^{n \times d} \times L_{\pi(\cdot)}^{2}\left(\mathbb{R}^{n}\right) \\
& \times \mathbb{R}^{m} \times \mathbb{R}^{n} \times \mathbb{R}^{n \times d} \times L_{\pi(\cdot)}^{2}\left(\mathbb{R}^{n}\right) \times \mathbf{E} \longrightarrow \mathbb{R}^{m}, \\
f & : \Omega \times[0, T] \times \mathbb{R}^{m} \times \mathbb{R}^{n} \times \mathbb{R}^{n \times d} \times L_{\pi(\cdot)}^{2}\left(\mathbb{R}^{n}\right) \\
& \times \mathbb{R}^{m} \times \mathbb{R}^{n} \times \mathbb{R}^{n \times d} \times L_{\pi(\cdot)}^{2}\left(\mathbb{R}^{n}\right) \longrightarrow \mathbb{R}^{n}, \\
\psi & : \mathbb{R}^{n} \longrightarrow \mathbb{R}^{m} .
\end{aligned}
$$

Given an $n \times m$ full-rank matrix $H$, let us introduce some notations

$$
\begin{aligned}
& \zeta=\left(\begin{array}{l}
x \\
y \\
z \\
r
\end{array}\right), \quad \zeta^{\prime}=\left(\begin{array}{l}
x^{\prime} \\
y^{\prime} \\
z^{\prime} \\
r^{\prime}
\end{array}\right), \\
& A\left(t, \zeta, \zeta^{\prime}\right)=\left(\begin{array}{c}
-H^{T} f \\
H b \\
H \sigma \\
H \gamma
\end{array}\right)\left(t, \zeta, \zeta^{\prime}\right),
\end{aligned}
$$

where $H \sigma=\left(H \sigma_{1} \cdots H \sigma_{d}\right)$. Assume that

(A3) for each $\zeta, \zeta^{\prime} \in \mathbb{R}^{m} \times \mathbb{R}^{n} \times \mathbb{R}^{n \times d} \times L_{\pi(\cdot)}^{2}\left(\mathbb{R}^{n}\right), A\left(\cdot, \zeta, \zeta^{\prime}\right)$ is an $\mathscr{F}_{t}$-measurable process defined on $[0, T]$ with $A(\cdot, 0,0) \in M^{2}\left(0, T ; \mathbb{R}^{m} \times \mathbb{R}^{n} \times \mathbb{R}^{n \times d}\right) \times F_{N}^{2}\left(0, T ; \mathbb{R}^{n}\right) ;$

(A4) $A\left(t, \zeta, \zeta^{\prime}\right)$ and $\psi(y)$ satisfy Lipschitz conditions: there exists a constant $k>0$, such that

$$
\begin{gathered}
\left|A\left(t, \zeta, \zeta^{\prime}\right)-A\left(t, \bar{\zeta}, \bar{\zeta}^{\prime}\right)\right| \leq k\left(|\zeta-\bar{\zeta}|+\left|\zeta^{\prime}-\bar{\zeta}^{\prime}\right|\right), \\
\forall \zeta=(x, y, z, r)^{T}, \quad \zeta^{\prime}=\left(x^{\prime}, y^{\prime}, z^{\prime}, r^{\prime}\right)^{T}, \\
\bar{\zeta}=(\bar{x}, \bar{y}, \bar{z}, \bar{r})^{T}, \\
\bar{\zeta}^{\prime}=\left(\bar{x}^{\prime}, \bar{y}^{\prime}, \bar{z}^{\prime}, \bar{r}^{\prime}\right)^{T} \in \mathbb{R}^{m} \times \mathbb{R}^{n} \times \mathbb{R}^{n \times d} \times L_{\pi(\cdot)}^{2}\left(\mathbb{R}^{n}\right), \\
\forall t \in[0, T], \\
|\psi(y)-\psi(\bar{y})| \leq k|y-\bar{y}|, \quad \forall y, \bar{y} \in \mathbb{R}^{n} ;
\end{gathered}
$$

(A5) $A\left(t, \zeta, \zeta^{\prime}\right)$ and $\psi(y)$ satisfy monotonic conditions:

$$
\begin{gathered}
\left\langle A\left(t, \zeta, \zeta^{\prime}\right)-A\left(t, \bar{\zeta}, \bar{\zeta}^{\prime}\right), \zeta-\bar{\zeta}\right\rangle \\
\leq-\mu_{1}|H(x-\bar{x})|^{2} \\
-\mu_{2}\left(\left|H^{T}(y-\bar{y})\right|^{2}+\left|H^{T}(z-\bar{z})\right|^{2}\right. \\
\left.+\left\|H^{T}(r-\bar{r})\right\|^{2}\right), \\
\forall \zeta=(x, y, z, r)^{T}, \quad \zeta^{\prime}=\left(x^{\prime}, y^{\prime}, z^{\prime}, r^{\prime}\right)^{T}, \\
\bar{\zeta}=(\bar{x}, \bar{y}, \bar{z}, \bar{r})^{T}, \\
\bar{\zeta}^{\prime}=\left(\bar{x}^{\prime}, \bar{y}^{\prime}, \bar{z}^{\prime}, \bar{r}^{\prime}\right)^{T} \in \mathbb{R}^{m} \times \mathbb{R}^{n} \times \mathbb{R}^{n \times d} \times L_{\pi(\cdot)}^{2}\left(\mathbb{R}^{n}\right), \\
\langle\psi(y)-\psi(\bar{y}), y-\bar{y}\rangle \leq-\beta_{2}\left|H^{T}(y-\bar{y})\right|^{2}, \quad \forall y, \bar{y} \in \mathbb{R}^{n},
\end{gathered}
$$

where $\mu_{1}, \mu_{2}$, and $\beta_{2}$ are given nonnegative constants with $\mu_{1}+\mu_{2}>0, \mu_{1}+\beta_{2}>0$. Moreover we have $\mu_{1}>0$ (resp., $\mu_{2}>0, \beta_{2}>0$ ) when $m<n$ (resp., $m>n)$.

By similar arguments of $\mathrm{Yu}$ and Ji [14], Wang and $\mathrm{Yu}$ [19], and Min et al. [25], we have the following existence and uniqueness theorem.

Theorem 7. One assumes that (A3), (A4), and (A5) hold. Then MF-FBSDEJ (68) has a unique solution $(x(t), y(t), z(t)$, $r(t, \cdot)) \in M^{2}\left(0, T ; \mathbb{R}^{m} \times \mathbb{R}^{n} \times \mathbb{R}^{n \times d}\right) \times F_{N}^{2}\left(0, T ; \mathbb{R}^{n}\right)$.

\section{Conclusion}

In this paper, we investigate a new differential game problem of mean-field BSDE with jump (MF-BSDEJ). Compared with the previous literature, our game systems are mean-field BSDE with jump and are under the framework of partial information. We established a maximum principle and a verification theorem for an equilibrium point of nonzero sum differential games. We also give a partial information linearquadratic (LQ) game as example to show the applications of our theoretical results.

The subject issue studied in this paper possesses fine generality. Firstly, the mean-field BSDEJ game system covers many systems as its particular case. For example, if we drop the terms on jump or mean-field or both of them, then the MF-BSDEJ can be reduced to MF-BSDE or BSDEJ or BSDE. Secondly, if we suppose that $\mathscr{E}_{t}=\mathscr{F}_{t}$, for all $t \in[0, T]$, all the results are reduced to the case of full information. Thirdly, if the present nonzero sum stochastic differential game has only one player, the game problem is reduced to some related optimal control. Particularly, our results are a partial extension to differential games of full information BSDEs [15] and partial information BSDEs [19]. Finally, since many optimization and game problems in finance and 
economics can be associated with mean-field BSDE with jump, the outcomes of this paper can be widely applied in these areas.

\section{Conflict of Interests}

The authors declare that there is no conflict of interests regarding the publication of this paper.

\section{Acknowledgments}

The authors would like to thank the editors and the reviewers for their constructive comments and suggestions which helped them to improve this paper. This work was supported by the National Natural Science Foundation of China (Grant nos. 11371226, 11071145, 11301298, and 11231005), the Foundation for Innovative Research Groups of National Natural Science Foundation of China (Grant no. 11221061), the 111 Project (Grant no. B12023), and the Development Project of Shandong Province Science and Technology (Grant no. 2012GGB01241).

\section{References}

[1] R. Isaacs, "Differential Games, Parts 1-4," The Rand Corpration, Research Memorandums Nos. RM-1391, RM-1411, RM-1486, 1954-1955.

[2] T. Basar and G. J. Olsder, Dynamic Noncooperative Game Theory, vol. 160 of Mathematics in Science and Engineering, Academic Press, London, UK, 1982.

[3] D. W. K. Yeung and L. A. Petrosyan, Cooperative Stochastic Differential Games, Springer, New York, NY, USA, 2005.

[4] L. D. Berkovitz, "Necessary conditions for optimal strategies in a class of differential games and control problems," SIAM Journal on Control, vol. 5, no. 1, pp. 1-24, 1967.

[5] W. H. Fleming, "The convergence problem for differential games," Journal of Mathematical Analysis and Applications, vol. 3, pp. 102-116, 1961.

[6] R. J. Elliott and N. J. Kalton, "The existence of value in differential games," Bulletin of the American Mathematical Society, vol. 126, pp. 1-67, 1972.

[7] A. Friedman, Differential Games, Wiley-Interscience, New York, NY, USA, 1971.

[8] P. Varaiya, "N-player stochastic differential games," SIAM Journal on Control and Optimization, vol. 14, no. 3, pp. 538-545, 1976.

[9] R. J. Elliott and M. H. A. Davis, "Optimal play in a stochastic differential game," SIAM Journal on Control and Optimization, vol. 19, no. 4, pp. 543-554, 1981.

[10] S. Hamadène, "Nonzero sum linear-quadratic stochastic differential games and backward-forward equations," Stochastic Analysis and Applications, vol. 17, no. 1, pp. 117-130, 1999.

[11] S. Hamadène, J. P. Lepeltier, and S. Peng, "BSDE with continuous coefficients and applications to Markovian nonzero sum stochastic differential games," in Pitman Research Notes Mathematics Series: Vol. 364. Backward Stochastic Differential Equations, N. El Karoui and L. Mazliak, Eds., pp. 115-128, Longman, Harlow, UK, 1997.
[12] E. Altman, "Applications of dynamic games in queues", in Advances in Dynamic Games, vol. 7, pp. 309-342, Birkhauser, Boston, Mass, USA, 2005.

[13] Z. Wu and Z. Y. Yu, "Linear quadratic nonzero-sum differential games with random jumps," Applied Mathematics and Mechanics, vol. 26, no. 8, pp. 1034-1039, 2005.

[14] Z. Yu and S. Ji, "Linear-quadratic nonzero-sum differential game of backward stochastic differential equations," in Proceedings of the 27th Chinese Control Conference (CCC '08), pp. 562566, Kunming, China, July 2008.

[15] G. Wang and Z. Yu, "A Pontryagin's maximum principle for non-zero sum differential games of BSDEs with applications," IEEE Transactions on Automatic Control, vol. 55, no. 7, pp. 1742 $1747,2010$.

[16] T. T. K. An and B. Øksendal, "Maximum principle for stochastic differential games with partial information," Journal of Optimization Theory and Applications, vol. 139, no. 3, pp. 463-483, 2008.

[17] T. T. K. An and B. Øksendal, "A maximum principle for stochastic differential games with $g$-expectations and partial information," Stochastics, vol. 84, no. 2-3, pp. 137-155, 2012.

[18] T. T. K. An, B. Øksendal, and Y. Y. Okur, "A Malliavin calculus approach to general stochastic differential games with partial information," in Malliavin Calculus and Stochastic Analysis, vol. 34 of Springer Proceedings in Mathematics \& Statistics, pp. 489510, Springer, Berlin, Germany.

[19] G. Wang and Z. Yu, "A partial information non-zero sum differential game of backward stochastic differential equations with applications," Automatica, vol. 48, no. 2, pp. 342-352, 2012.

[20] Q. Meng and M. Tang, "Stochastic differential games of fully coupled forward-backward stochastic systems under partial information," in Proceedings of the 29th Chinese Control Conference (CCC '10), pp. 1150-1155, Beijing, China, July 2010.

[21] E. C. M. Hui and H. Xiao, "Differential games of partial information forward-backward doubly SDE and applications," ESAIM: Control, Optimisation and Calculus of Variations, vol. 20, no. 1, pp. 78-94, 2014.

[22] B. Øksendal and A. Sulem, "Forward-backward stochastic differential games and stochastic control under model uncertainty," Journal of Optimization Theory and Applications, vol. 161, no. 1, pp. 22-55, 2014.

[23] Y. Shen and T. K. Siu, "The maximum principle for a jumpdiffusion mean-field model and its application to the meanvariance problem," Nonlinear Analysis, Theory, Methods and Applications, vol. 86, pp. 58-73, 2013.

[24] J. Xiong, An Introduction to Stochastic Filtering Theory, Oxford University Press, London, UK, 2008.

[25] H. Min, Y. Peng, and Y. Qin, "Fully coupled mean-field forwardbackward stochastic differential equations and stochastic maximum principle," Abstract and Applied Analysis, vol. 2014, Article ID 839467, 15 pages, 2014. 


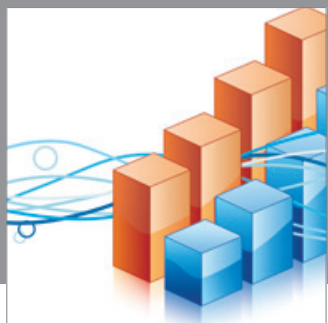

Advances in

Operations Research

mansans

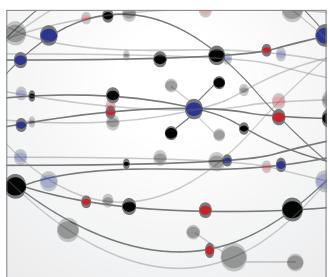

The Scientific World Journal
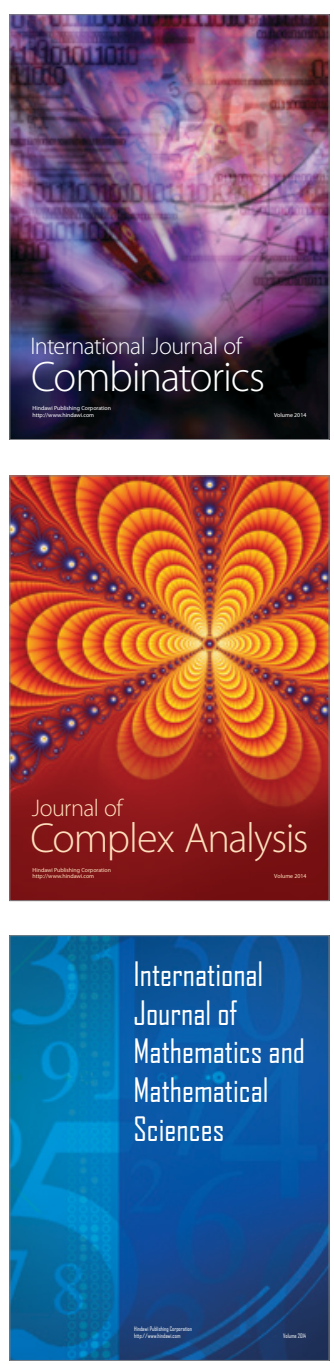
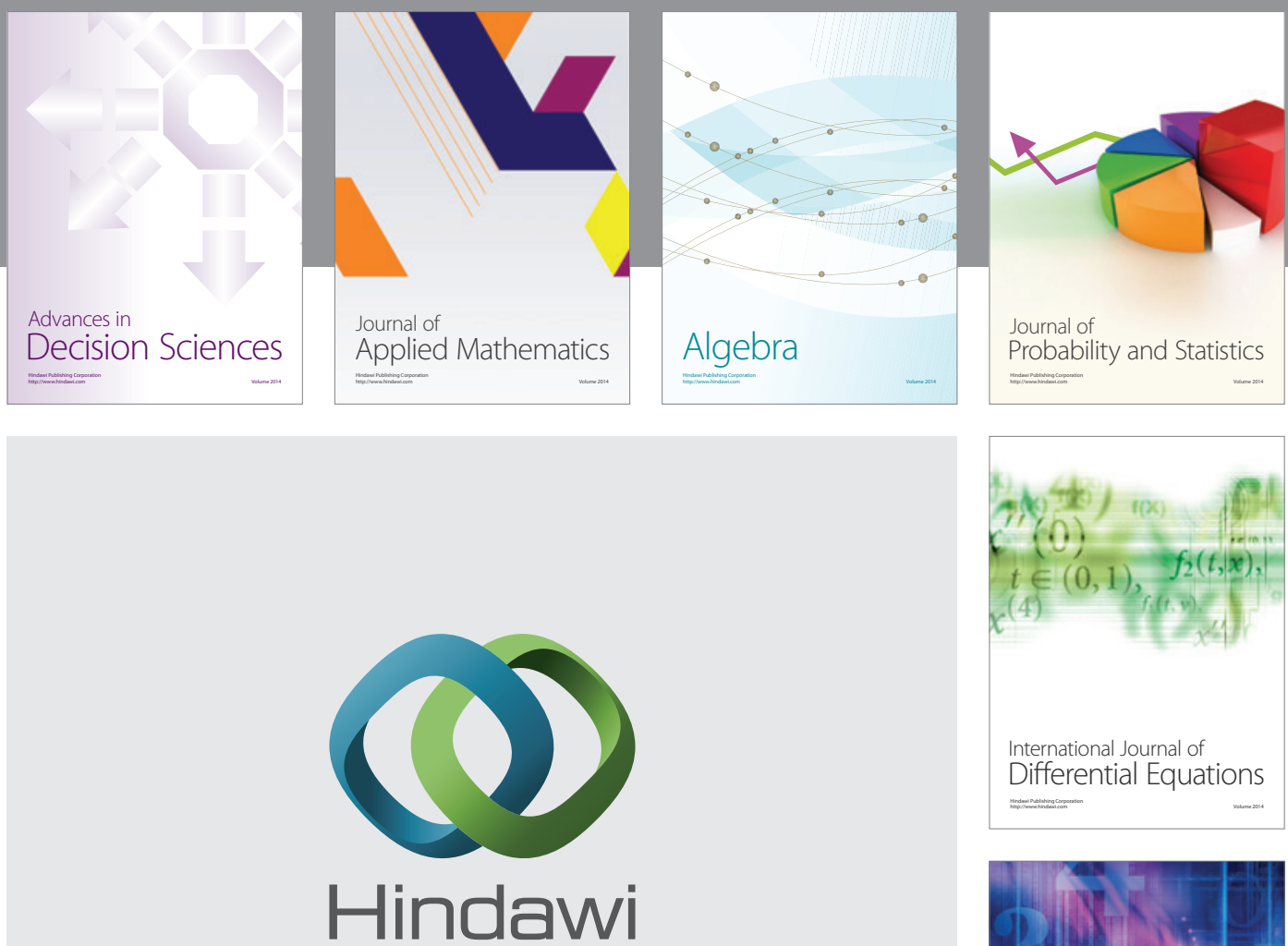

Submit your manuscripts at http://www.hindawi.com
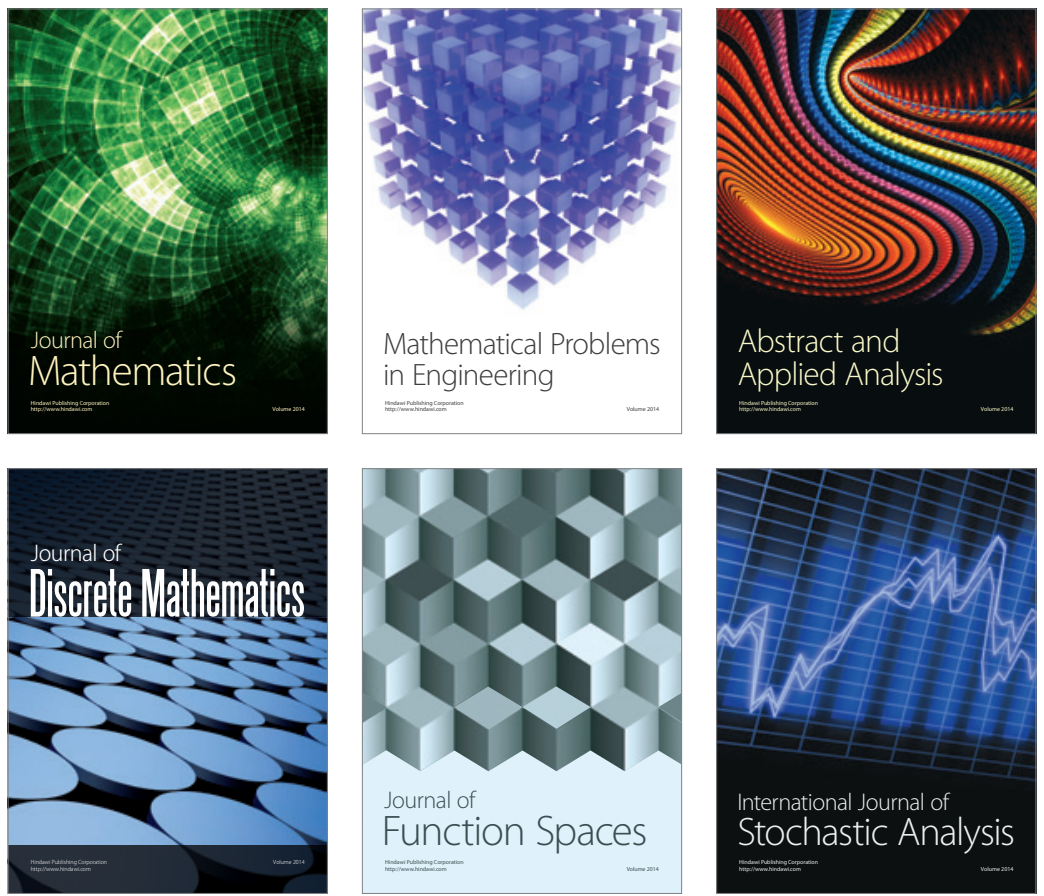

Journal of

Function Spaces

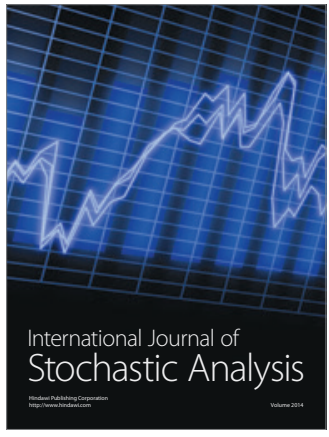

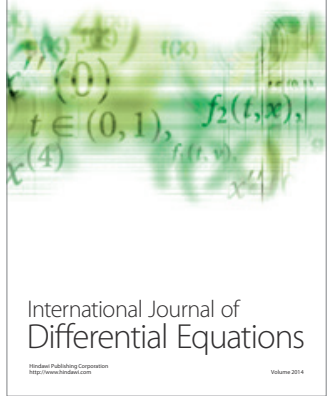
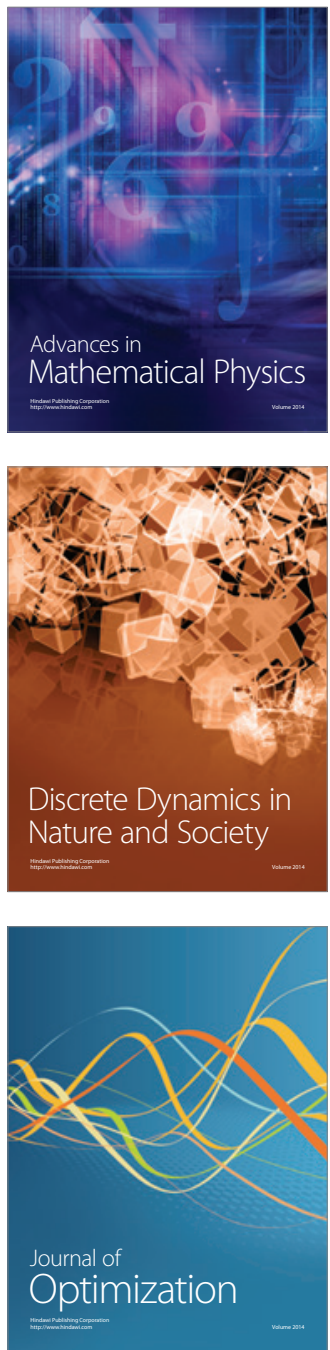